\title{
The young intermediate-mass stellar object AFGL 490 - A disk surrounded by a cold envelope ${ }^{\star}$
}

\author{
K. Schreyer ${ }^{1}$, Th. Henning ${ }^{2}$, F. F. S. van der Tak ${ }^{3}$, A. M. S. Boonman ${ }^{4}$, and E.F. van Dishoeck ${ }^{4}$ \\ 1 Astrophysikalisches Institut und Universitäts-Sternwarte (AIU), Schillergäßchen 2-3, 07745 Jena, Germany \\ 2 Max-Planck-Institut für Astronomie, Königstuhl 17, 69117 Heidelberg, Germany \\ 3 Max-Planck-Institut für Radioastronomie, Auf dem Hügel 69, 53121 Bonn, Germany \\ ${ }^{4}$ Leiden Observatory, PO Box 9513, 2300 RA Leiden, The Netherlands
}

Received 16 January 2002 / Accepted 5 August 2002

\begin{abstract}
AFGL 490 is a key target of the class of deeply embedded intermediate-mass young stellar objects in a transition stage to Herbig Be stars $\left(L=2.2-4.0 \times 10^{3} L_{\odot}\right)$. In this paper, we present a comprehensive set of single-dish line data which characterize the envelope of the source. In addition, observations of CS $J=2 \rightarrow 1$ and the corresponding continuum at $97.98 \mathrm{GHz}$ have been obtained with the Plateau de Bure $(\mathrm{PdB})$ interferometer, which are sensitive to the small-scale structure around the stellar source. The PdB line data show a bar-like elongated gas structure of $22000 \mathrm{AU} \times 6000 \mathrm{AU}$ size with a position angle of $\approx-45^{\circ}$. This bar represents the flattened inner envelope surrounding a disk-like structure (radius $\leq 500 \mathrm{AU}$ ) for which we find evidence very close to the young B star. Due to strong (self-)absorption in the velocity range $v_{\mathrm{lsr}}=-12.5 \ldots-15 \mathrm{~km} \mathrm{~s}^{-1}$, only the outer line wings can be used to study the gas motion. Maps of the integrated red and blue line wing emission show two well-separated gas blobs around AFGL 490, which are interpreted as a disk. The $3 \mathrm{~mm}$ continuum interferometer map shows a point source at the position of AFGL 490 with a flux of $240 \mathrm{mJy}$. This flux translates into a total mass of 3-6 $M_{\odot}$ of the disk which is comparable to the stellar mass of about $8 M_{\odot}$. This configuration is unstable and will disappear in $10^{3}-10^{4}$ years due to gravitational instabilities. Photometric data from ISOPHOT and spectroscopic data from ISO-SWS have been obtained. Together with submillimetre continuum data a very complete spectral energy distribution of the envelope could be compiled. Analysis of the data shows that the central region of AFGL 490 has a steeper density gradient compared with the outer molecular envelope. All data clearly point to a low temperature (25-35 K) of this envelope. To determine the chemical state of the object, we determined the abundances of 13 molecules towards AFGL 490. The molecular line and ISO-SWS data are used to derive the gas-solid abundance ratios for $\mathrm{H}_{2} \mathrm{O}, \mathrm{CO}$, and $\mathrm{CO}_{2}$. The chemical results, such as the relatively low gas-to-solid ratios and the low $\mathrm{CH}_{3} \mathrm{OH}$ excitation, emphasize the presence of a cold molecular envelope. We found evidence for other outflow systems in the envelope around AFGL 490. Red-shifted and blue-shifted gas blobs with a separation of about 20000 AU were detected. Their centre is located roughly 3 " to the south of AFGL 490, and their morphology implies that a deeply embedded low-mass object drives a jet which enters the denser envelope material at such a large distance. Two further outflow systems in the close neighbourhood of AFGL 490 could be identified. All these data point to the formation of a group of low-mass stars around AFGL 490. It is very remarkable that these outflows do not influence the global physical and chemical structure of the envelope.
\end{abstract}

Key words. ISM: clouds - ISM: individual objects: AFGL 490 - ISM: jets and outflows - ISM: molecules - stars: formation

\section{Introduction}

Our detailed knowledge about the circumstellar structure of embedded low-mass young stellar objects (YSOs) and highmass YSOs has increased very rapidly during recent years due to the advent of array receivers on single-dish telescopes and the application of millimetre interferometry (see, e.g.,

Send offprint requests to: $\mathrm{K}$. Schreyer,

e-mail: martin@astro.uni-jena.de

* Based on observations with ISO, an ESA project with instruments funded by ESA Member States (especially the PI countries: France, Germany, The Netherlands and the UK) with the participation of ISAS and NASA.
Evans 1999; Churchwell 1999). This is less the case for intermediate-mass YSOs, which are of special interest as the link between the low- and high-mass stars and the precursors of Vega-like systems (Di Francesco et al. 1997; Mannings \& Sargent 1997, 2000). Although there is firm evidence for the presence of Keplerian disks around very young low-mass YSOs and T Tauri stars (e.g. Dutrey et al. 1996; Koerner \& Sargent 1995; McCaughrean et al. 2000), interferometric evidence for disks around intermediate-mass stars only exists for a few already optically visible Herbig Ae/Be stars (Mannings \& Sargent 1997; Natta et al. 2000), but not for deeply embedded intermediate-mass YSOs. Interesting exceptions are 
the recent detections of disks around the massive B-type protostars IRAS 20126+4104 (Cesaroni et al. 1999; Zhang et al. 1998), and G 192.16-3.82 (Shepherd et al. 2001). In fact, disks around such objects may be much more massive and extended compared with their low-mass counterparts.

In order to test theories of the physical and chemical structure of YSOs, it is important to select relatively isolated objects which are not influenced by the formation of other massive and intermediate-mass stars in their close neighborhood (Grady et al. 1999a,b; Natta et al. 2000). In this respect, AFGL 490 is a prominent member of the class of intermediatemass YSOs and it seems to be an excellent nearby (distance $d \approx$ $1 \mathrm{kpc}$ ) candidate for such a study. AFGL 490 is deeply embedded in dense molecular gas and it is already well investigated by previous studies (e.g. Harvey et al. 1979; Lada \& Harvey 1981; Henning et al. 1990; Chini et al. 1991; Mundy \& Adelman 1988; Mitchell et al. 1992, 1995; van der Tak et al. 2000b). Earlier interferometry measurements obtained with the Berkeley Illinois Maryland Association (BIMA) interferometer (spatial resolution 7.8" $\times 7.2^{\prime \prime}$, Mundy \& Adelman 1988) and the Nobeyama interferometer (spatial resolution 3.3" $\times 5.5^{\prime \prime}$, Nakamura et al. 1991) suggest the existence of a large disklike structure around this object.

In this paper, we present a comprehensive data set of singledish and interferometer line and continuum observations as well as measurements obtained with ISOPHOT and ISO-SWS. The aim of this paper is better physical understanding of the source and its close environment. We mapped the envelope in $\mathrm{CS} J=7 \rightarrow 6,5 \rightarrow 4,3 \rightarrow 2,2 \rightarrow 1$, and in $\mathrm{C}^{18} \mathrm{O} J=2 \rightarrow 1$. Several additional spectral line settings were taken at the source position to tightly constrain the density structure. A wide set of continuum data including ISOPHOT measurements have been used to compile a very complete spectral energy distribution (SED). Furthermore, we studied the source in CS $J=2 \rightarrow 1$ and the corresponding continuum using the Plateau de Bure (PdB) interferometer to search for the presence of a Keplerian disk and to study the structure of the associated outflow close to the embedded intermediate-mass star which should be in an earlier evolutionary phase than the Herbig Ae/Be stars. The PdB data have much better quality than previous interferometry observations of AFGL 490.

Performing one-dimensional (1-D) continuum radiation transfer (RT) calculations using the RT code developed by Men'shchikov \& Henning (1997) and improved by Manske et al. (1998), we derive an envelope model which is compared to the model calculations performed by van der Tak et al. (2000b) using both continuum and line data. The interferometry data together with the envelope model allow us to search for the presence of a disk.

In order to obtain a more complete picture of the chemical composition towards AFGL 490, we determine both the gas phase abundances of different species coming from the comprehensive submillimeter molecular emission line study as well as the solid-phase composition derived from absorption features present in ISO-SWS observations. The gas/solid ratios, abundances, and excitation of such molecules as $\mathrm{CH}_{3} \mathrm{OH}$ are useful tracers of the temperature structure of the molecular envelope and thus its evolutionary state.
An additional goal of this work is the comparison of AFGL 490 with other intermediate-mass young objects such as NGC 2264 IRS1 (Schreyer et al. 1997, 2002), LkH $\alpha 225$ (van den Ancker 2000), G 192.16-3.82 (Shepherd et al. 2001), and Orion BN-KL (see Gezari et al. 1998: recalculation of the luminosity).

\section{General properties of AFGL 490}

Joyce et al. (1977) detected a strong $2 \mu \mathrm{m}$ infrared source at the position $\operatorname{RA}(B 1950)=03^{\mathrm{h}} 23^{\mathrm{m}} 38.8^{\mathrm{s}}$ and $\operatorname{Dec}(B 1950)=$ $58^{\circ} 36^{\prime} 39^{\prime \prime}$. Later, Campbell et al. (1989) corrected this position to $\operatorname{RA}(B 1950)=03^{\mathrm{h}} 23^{\mathrm{m}} 38.996^{\mathrm{s}}$ and $\operatorname{Dec}(B 1950)=$ $58^{\circ} 36^{\prime} 34.79^{\prime \prime}\left( \pm 0.3^{\prime \prime}\right)$. Whereas optical images show only a diffuse nebulosity at this position, AFGL 490 is a luminous source in the near-infrared (e.g., Campbell et al. 1986; Minchin et al. 1991; Haas et al. 1992; Hodapp 1994) surrounded by a couple of low-luminosity objects with projected distances between 5500 and $43000 \mathrm{AU}$.

The spectral energy distribution (SED) of AFGL 490 is well known with a spectral coverage from the visible to the radio region (Gear et al. 1986; Mundy \& Adelman 1988; Campbell et al. 1989; Chini et al. 1991). The total luminosity of AFGL 490 was determined to be $1.4 \times 10^{3}$ to $2.2 \times 10^{3} L_{\odot}$ applying a distance of $900 \mathrm{pc}$ which implies a spectral type of B2 to B3 (Harvey et al. 1979; Mozurkewich et al. 1986; Henning et al. 1990; Chini et al. 1991). However, Snell et al. (1984) noted that this distance is somewhat uncertain and used a value of $1.0( \pm 0.5) \mathrm{kpc}$. Furthermore, the kinematically estimated distance (Brand 1986; Brand \& Blitz 1993) is 1.2( \pm 0.2$) \mathrm{kpc}$ using $v_{\mathrm{lsr}}=-13.4( \pm 2) \mathrm{km} \mathrm{s}^{-1}$. This paper adopts a distance of $1 \mathrm{kpc}$.

We cannot exclude that part of the luminosity is produced by accretion. However, massive objects close to the ZAMS have luminosities that are dominated by contraction luminosities (Stahler et al. 2000). As soon as hydrogen ignites, further contraction stops and the star joins the ZAMS. Nevertheless, a time-dependent unstable accretion process could explain the NIR and radio variability observed by Hoare (2001).

Taking all infrared and radio observations together, AFGL 490 seems to be a luminous source with typical properties of a Becklin-Neugebauer-type object (Simon et al. 1981a; Scoville et al. 1983; Henning et al. 1990) with broad and strong $\operatorname{Br} \alpha$ and $\operatorname{Br} \gamma$ lines (Simon et al. 1979; Thompson \& Tokunaga 1979; Simon et al. 1981b; Bunn et al. 1995) as well as a weak continuum flux at wavelengths $\lambda \geq 1 \mathrm{~cm}$ (Simon et al. 1981a, 1983).

Based on $1.3 \mathrm{~cm}$ VLA and $\operatorname{Br} \gamma$, Pf $\gamma$ and $\operatorname{Br} \alpha$ infrared observations, Simon et al. (1983) estimated a maximum extent of the ionized region of $\leq 100 \mathrm{AU}$ and a mass loss rate of $10^{-6} M_{\odot} \mathrm{yr}^{-1}$. More recent measurements of $\operatorname{Br} \alpha$ and $\operatorname{Br} \gamma$ by Bunn et al. (1995) imply the presence of an accelerating stellar wind and show no evidence for low-velocity and less optically thick material around AFGL 490.

Line observations (e.g., Kawabe et al. 1984; Snell et al. 1984; Plume et al. 1992; Mitchell et al. 1992, 1995; Hasegawa \& Mitchell 1995; van der Tak et al. 2000b) indicate that AFGL 490 is located in a dense and very compact envelope. The object is associated with a poorly collimated high-velocity 
Table 1. Beam efficiencies at the JCMT during different observing runs.

\begin{tabular}{|c|c|c|c|c|}
\hline \multirow[t]{3}{*}{ observing run } & \multicolumn{3}{|c|}{ beam efficiencies } & \multirow{3}{*}{$\begin{array}{l}\text { number } \\
\text { of } \\
\text { spectra }\end{array}$} \\
\hline & RxA2: & RxB3i: & $\mathrm{RxC2:}$ & \\
\hline & $230 \mathrm{GHz}$ & $345 \mathrm{GHz}$ & $460 \mathrm{GHz}$ & \\
\hline February 1994 & 0.53 & 0.45 & 0.35 & 1 \\
\hline June 1994 & 0.72 & 0.60 & 0.42 & 6 \\
\hline October 1995 & 0.69 & $\ldots$ & $\ldots$ & 5 \\
\hline May 1996 & $\ldots$ & 0.60 & $\ldots$ & 1 \\
\hline October 1996 & $\ldots$ & $\ldots$ & 0.53 & 2 \\
\hline December 1996 & $\ldots$ & $\ldots$ & 0.53 & 1 \\
\hline June 1997 & 0.64 & 0.63 & $\ldots$ & 4 \\
\hline November 1997 & $\ldots$ & 0.63 & $\ldots$ & 1 \\
\hline January 1998 & 0.69 & 0.64 & 0.53 & 2 \\
\hline
\end{tabular}

bipolar outflow in the direction northeast-southwest (e.g., Lada \& Harvey 1981; Snell et al. 1984; Kawabe et al. 1984; Mitchell et al. 1992). Mitchell et al. (1995) concluded that gas in the high-velocity outflow is organized into numerous dense clumps with masses of 0.01 to $0.5 M_{\odot}$ and velocities ranging from few $\mathrm{km} \mathrm{s}^{-1}$ to $40 \mathrm{~km} \mathrm{~s}^{-1}$. An additional high-resolution ${ }^{13} \mathrm{CO}$ absorption spectrum in the $M$ band $(4.6 \mu \mathrm{m})$ and $\mathrm{CO} J=6 \rightarrow 5$ observations by Mitchell et al. (1995) indicate the presence of a warm gas component with kinetic temperatures of $T_{\text {kin }} \geq 100 \mathrm{~K}$ in a radius of $\approx 6000 \mathrm{AU}$ and a ${ }^{13} \mathrm{CO}$ column density of $N_{\text {warm }}=$ $6.9 \times 10^{16} \mathrm{~cm}^{-2}$. The cold gas component in the ${ }^{13} \mathrm{CO}$ absorption spectrum $\left(T_{\text {kin }}=24 \mathrm{~K}\right)$ is comparable $N_{\text {cold }}=5.6 \times$ $10^{16} \mathrm{~cm}^{-2}$.

A number of previous high-resolution observations suggest the presence of a circumstellar disk around the central object AFGL 490. Interferometric continuum measurements by Mundy \& Adelman (1988, resolution: 2.2" $\left.\times 2.1^{\prime \prime}\right)$ show AFGL 490 to be centered in an elongated continuum source with an extent of $2600 \times 1500 \mathrm{AU}$. In addition, there is evidence for the presence of an even larger elongated ${ }^{13} \mathrm{CO} J=$ $1 \rightarrow 0$ gas structure (resolution: $7.8^{\prime \prime} \times 7.2^{\prime \prime}$ ) with an extent of $45000 \times 14000 \mathrm{AU}$ and the same orientation (southeastnorthwest) which is perpendicular to the high-velocity outflow. Whereas Kawabe et al. (1984) discussed the presence of an expanding gas torus around AFGL 490, Nakamura et al. (1991, CS $J=2 \rightarrow 1$, resolution: $\left.3.3^{\prime \prime} \times 5.5^{\prime \prime}\right)$ found some evidence for infall motion. However, the interpretation of the data remains ambiguous. Our PdB data have better quality and we will show evidence for self-absorption in the CS $J=2 \rightarrow 1$ line which changes previous interpretations of the data. We find strong evidence for a compact millimetre source which is very probably a disk-like structure around the stellar object.

Various studies ranging from the optical to the near-infrared wavelength range (Campbell et al. 1989; Persson et al. 1988; Yamashita et al. 1989; Minchin et al. 1991; Hoare et al. 1996) also point to the presence of a disk around AFGL 490 with a diameter of about $1000 \mathrm{AU}$ based on polarization measurements and elongated infrared emission. However, the value

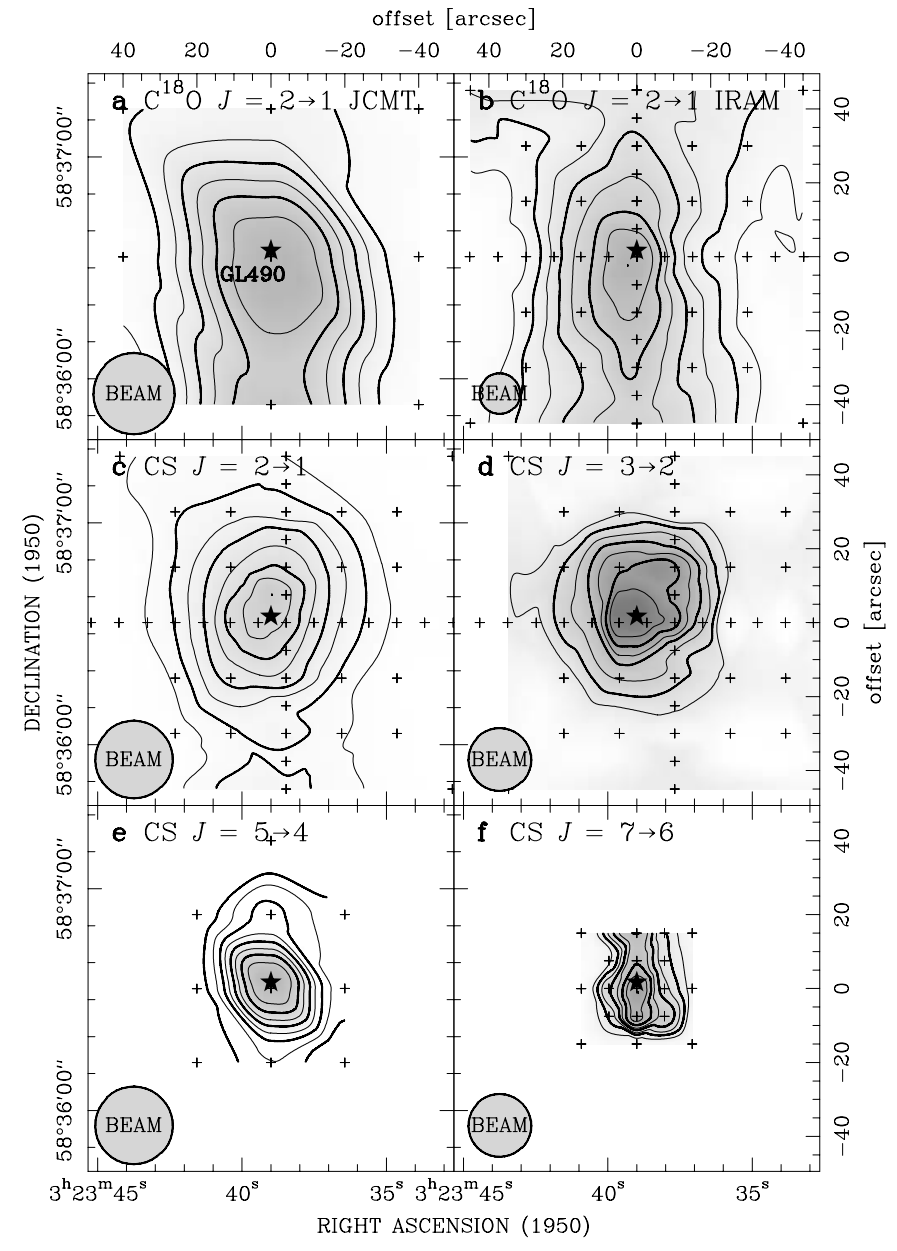

Fig. 1. a)-f) Contour plots of the total integrated line emission in a certain velocity range $v_{\mathrm{lsr}}$ : a) $\mathrm{C}^{18} \mathrm{O} J=2 \rightarrow 1, v_{\mathrm{lsr}}=-15.5$ to $-10.0 \mathrm{~km} \mathrm{~s}^{-1}$ measured at JCMT (peak value $=23.7 \mathrm{~K} \mathrm{~km} \mathrm{~s}^{-1}$ ); b) $\mathrm{C}^{18} \mathrm{O} J=$ $2 \rightarrow 1, v_{\mathrm{lsr}}=-15.5$ to $-9.5 \mathrm{~km} \mathrm{~s}^{-1}$ measured at IRAM (peak value $=$ $62.3 \mathrm{~K} \mathrm{~km} \mathrm{~s}^{-1}$ ); c) CS $J=2 \rightarrow 1, v_{\mathrm{lsr}}=-15.5$ to $-10.0 \mathrm{~km} \mathrm{~s}^{-1}$ (peak value $\left.=32.4 \mathrm{~K} \mathrm{~km} \mathrm{~s}^{-1}\right)$; d) CS $J=3 \rightarrow 2, v_{\mathrm{lsr}}=-16.0$ to $-10.0 \mathrm{~km} \mathrm{~s}^{-1}$ (peak value $=24.8 \mathrm{~K} \mathrm{~km} \mathrm{~s}^{-1}$ ); e) CS $J=5 \rightarrow 4, v_{\mathrm{lsr}}=-17.0$ to $-9.23 \mathrm{~km} \mathrm{~s}^{-1}$ (peak value $=17.2 \mathrm{~K} \mathrm{~km} \mathrm{~s}^{-1}$ ); f) CS $J=7 \rightarrow 6$, $v_{\text {lsr }}=-15.6$ to $-10.15 \mathrm{~km} \mathrm{~s}^{-1}$ (peak value $=8.3 \mathrm{~K} \mathrm{~km} \mathrm{~s}^{-1}$ ). The contours are 30 to $90 \%$ of the peak values in steps of $10 \%$. The small crosses indicate the points of the measurements. The position of AFGL 490 is shown by the star in the centre of each figure.

of the inclination angle of the disk is very controversial. Whereas Campbell et al. (1989) assumed that optical images of AFGL 490 show only scattered light, Hodapp (1994) argues that direct optical light from the star is seen. The continuum measurements by Mundy \& Adelman (1988) indicate rather an edge-on location of the disk. In contrast to this, Mitchell et al. (1995) note that the short projected extent of the outflow lobes suggest a larger inclination angle. Nakamura et al. (1991) determined an inclination angle of $i=48^{\circ}-60^{\circ}$ by fitting a disk model to interferometric CS observations. Comparable results have been found by Minchin et al. (1991) using polarization measurements. 


\section{Observations}

\subsection{Observations with the JCMT}

The region around AFGL 490 was mapped in CS $J=5 \rightarrow 4$, $7 \rightarrow 6, \mathrm{C}^{18} \mathrm{O} J=2 \rightarrow 1$, and ${ }^{12} \mathrm{CO} J=3 \rightarrow 2$ with the James Clerk Maxwell Telescope (JCMT) ${ }^{1}$ on Mauna Kea, Hawaii, in June 1994. We used the facility receivers A2 (216-280 GHz), B3i (300-380 GHz), and C2 (450-500 GHz) as the frontends with the Digital Autocorrelation Spectrometer (DAS) as the backend. For mapping, the total bandwidths were $500 \mathrm{MHz}$ for $\mathrm{C}^{18} \mathrm{O} \mathrm{J}=2 \rightarrow 1$ and $250 \mathrm{MHz}$ for the CS transitions, centered at $v_{\text {lsr }}=-13.4 \mathrm{~km} \mathrm{~s}^{-1}$. The CS $J=7 \rightarrow 6$ line at $342.883 \mathrm{GHz}$ was placed in the lower sideband so that the ${ }^{12} \mathrm{CO} 3 \rightarrow 2$ line at $345.330 \mathrm{GHz}$ could be observed simultaneously in the upper sideband. The CS $5 \rightarrow 4$ setting at $244.936 \mathrm{GHz}$ was measured in the lower sideband. Furthermore, the CS $J=10 \rightarrow 9$ line $(489.751 \mathrm{GHz})$ was searched together with the $\mathrm{C}^{34} \mathrm{~S} J=$ $10 \rightarrow 9$ line $(481.916 \mathrm{GHz})$ in the opposite sideband.

The maps were obtained in position-switch mode with an off-position of $10^{\prime}$ to the east for the CS transitions and an off-position of $20^{\prime}$ for $\mathrm{C}^{18} \mathrm{O} J=2 \rightarrow 1$. The maps were sampled at half beamwidth intervals of $7.5^{\prime \prime}$ for the CS $J=7 \rightarrow 6$ transition, and at $15^{\prime \prime}$ spacing for the $220 \mathrm{GHz}$ transitions. For CS $J=5 \rightarrow 4$ and $\mathrm{C}^{18} \mathrm{O}$, we used an on+off total integration time of $5 \mathrm{~min}$. For the CS $J=7 \rightarrow 6$ transition $8 \mathrm{~min}$ were used.

The telescope half power beamwidth covered $12^{\prime \prime}$ for the $492 \mathrm{GHz}$ window, $15^{\prime \prime}$ for $345 \mathrm{GHz}$, and $21^{\prime \prime}$ for the $220 \mathrm{GHz}$ band. The main beam efficiencies $\eta_{\mathrm{mb}}$ varied over the different observing runs. Table 1 compiles the beam efficiencies which were determined from planets and standard sources. The line intensities were calibrated by the chopperwheel method (Kutner \& Ulich 1981) to get line strengths on the antenna temperature $\left(T_{\mathrm{A}}^{*}\right)$ scale. The main beam temperature is defined by $T_{\mathrm{mb}}=T_{\mathrm{A}}^{*} / \eta_{\mathrm{mb}}$. The pointing was checked every two to three hours on the source OMC 1, and was found to be accurate to better than $3^{\prime \prime}$.

In addition, we measured several spectral line settings at the position of AFGL 490 ([0, 0] map position) which are summarized in Table 2. These measurements were performed between February 1994 and January 1998. The observing parameters of these measurements are also compiled in Table 2. Beam switching was only used for higher-excitation lines of less abundant molecules with $3^{\prime}$ in azimuth. Most of the other transitions were observed by position-switching $10^{\prime}$ to the east.

Based on the large time interval in which the measurements of the molecular lines were done, we found some differences of the line intensities partly of the same transition or between various transitions of the same species. These differences are possibly caused by the varying atmospheric conditions, different spectral resolutions (see for example $\mathrm{C}^{18} \mathrm{O} J=2 \rightarrow 1$ ), or by different sideband gains.

\footnotetext{
${ }^{1}$ JCMT is operated by the Joint Astronomy Center on behalf of the Particle Physics and Astronomy Research Council of the UK, The Netherlands Organization for Scientific Research, and the National Research Council of Canada.
}

\subsection{Observations with the IRAM 30-m telescope}

In order to get more information about the excitation conditions and the line profiles of the lower CS transitions, we performed observations with the IRAM ${ }^{2} 30-m$ telescope on Pico Veleta, Spain, in October 1995. A region of $2^{\prime} \times 2^{\prime}$ around AFGL 490 was mapped simultaneously in CS $J=2 \rightarrow 1,3 \rightarrow 2$, and $\mathrm{C}^{18} \mathrm{O} J=2 \rightarrow 1$ with a spacing of $15^{\prime \prime}$ in the outer regions and $7.5^{\prime \prime}$ in the centre of the map. The beam sizes were $25^{\prime \prime}$ at $97.98 \mathrm{GHz}, 17^{\prime \prime}$ at $146.97 \mathrm{GHz}$, and $11^{\prime \prime}$ at $219.56 \mathrm{GHz}$. The backend consisted of an autocorrelator split into 3 subbands with a resolution of $40 \mathrm{kHz}$ for the CS lines and $80 \mathrm{kHz}$ for $\mathrm{C}^{18} \mathrm{O}$. The chopper-wheel method was applied to calibrate the spectra in values of $T_{\mathrm{A}}^{*}$. We changed the intensities of the spectra to main beam temperatures $T_{\mathrm{mb}}=T_{\mathrm{A}}^{*} / \eta_{\mathrm{mb}}$. For the values of $\eta_{\mathrm{mb}}$, we refer to the IRAM Newsletter, No. 18, 1994. The adopted main beam efficiencies were 0.72, 0.55, and 0.41 , respectively. The spectra were taken as on/off measurements with an off-position of $30^{\prime}$ to the east and with a total integration time of $2.25 \mathrm{~min}$. Pointing checks have been done about every $1.5 \mathrm{~h}$ depending on weather conditions and the elevation of the telescope. The typical pointing error was less than $5^{\prime \prime}$.

Caused by a non-perfect alignment of three receivers, the intensity peak positions in all three IRAM maps $\left(\mathrm{C}^{18} \mathrm{O} J=\right.$ $2 \rightarrow 1$, CS $J=2 \rightarrow 1$, and $3 \rightarrow 2$ ) do not quite coincide. We shifted the maps in CS $J=2 \rightarrow 1$ by $4.125^{\prime \prime}$ and in CS $J=3 \rightarrow 2$ by $10.3^{\prime \prime}$ to the west, so that the intensity peaks are roughly located at the position of AFGL 490.

\subsection{Observations with the Plateau de Bure interferometer}

With the PdB interferometer, we observed CS $J=2 \rightarrow 1$ as well as the corresponding continuum emission at $97.98 \mathrm{GHz}$. The interferometer properties are comprehensively described by Guilloteau et al. (1992). Successful observations were obtained with four 15-m antennas in July and October 1999 using the BC configuration (baseline lengths $21 \mathrm{~m}-254 \mathrm{~m}$ ). The phase reference centre of the measurements is $\operatorname{RA}(2000)=$ $03^{\mathrm{h}} 27^{\mathrm{m}} 38.55^{\mathrm{s}}$ and $\operatorname{Dec}(2000)=+58^{\circ} 46^{\prime} 59.80^{\prime \prime}$.

In order to resolve details of the velocity structure and to cover possible line wing emission, we used three correlator units each with $20 \mathrm{MHz}$ bandwidth placed close together with an overlap of 12/13 channels. For a higher frequency resolution, we applied a fourth correlator unit with a total bandwidth of $10 \mathrm{MHz}$ centered at the CS $J=2 \rightarrow 1$ line. The velocity resolutions are $0.24 \mathrm{~km} \mathrm{~s}^{-1}(=0.078 \mathrm{kHz})$ for the $20 \mathrm{MHz}$ unit and $0.12 \mathrm{~km} \mathrm{~s}^{-1}(=0.039 \mathrm{kHz})$ for the $10 \mathrm{MHz}$ unit. The CS $J=2 \rightarrow 1$ line was centered at $v_{\mathrm{lsr}}=-13.4 \mathrm{~km} \mathrm{~s}^{-1}$ in the LSB. The remaining two spectral correlator units, each with a bandwidth of $160 \mathrm{MHz}$, were used to measure the continuum at $97.98 \mathrm{GHz}$.

The band pass and phase calibration was performed on the objects 3C454.3 and 2145+067. An additional calibration of the phase and the amplitude was obtained by observing the objects $0355+508$ and $0224+671$ every $20 \mathrm{~min}$. For the final

\footnotetext{
2 Institut de Radio Astronomie Millimétrique.
} 
Table 2. Parameters of observed lines at the position of AFGL 490. Two-component fits are indicated with (a) and (b).

\begin{tabular}{|c|c|c|c|c|c|c|c|c|}
\hline Molecule & transition & $\begin{array}{c}v \\
{[\mathrm{MHz}]}\end{array}$ & $\begin{array}{l}T_{\mathrm{mb}} \\
{[\mathrm{K}]}\end{array}$ & $\begin{array}{l}\mathrm{rms} \\
{[\mathrm{K}]}\end{array}$ & $\begin{array}{r}\Delta v \\
{\left[\mathrm{~km} \mathrm{~s}^{-1}\right]}\end{array}$ & $\begin{array}{r}\int T_{\mathrm{mb}} \mathrm{d} v \\
{\left[\mathrm{~K} \mathrm{~km} \mathrm{~s}^{-1}\right]}\end{array}$ & $\begin{array}{r}\text { spec. resol. } \\
{\left[\mathrm{km} \mathrm{s}^{-1}\right]}\end{array}$ & telescope/date \\
\hline \multirow[t]{5}{*}{$\mathrm{CS}$} & $J=10 \rightarrow 9$ & 489751.0 & $\leq 2.0$ & 1.0 & - & - & 0.19 & JCMT/Jun. 94 \\
\hline & $J=7 \rightarrow 6$ & 342883.3 & 2.8 & 0.6 & 2.8 & 8.0 & 0.14 & JCMT/Jun. 94 \\
\hline & $J=5 \rightarrow 4$ & 244935.6 & 5.0 & 0.2 & 3.1 & 16.5 & 0.19 & JCMT/Jun. 94 \\
\hline & $J=3 \rightarrow 2$ & 146969.0 & 5.9 & 0.2 & 2.6 & 16.6 & 0.08 & IRAM/Sep. 95 \\
\hline & $J=2 \rightarrow 1$ & 97981.0 & 5.6 & 0.1 & 2.6 & 15.3 & 0.12 & IRAM/Sep. 95 \\
\hline \multirow[t]{3}{*}{$\mathrm{C}^{34} \mathrm{~S}$} & $J=10 \rightarrow 9$ & 481916.1 & $\leq 2.0$ & 1.0 & - & - & 0.19 & JCMT/Jun. 94 \\
\hline & $J=7 \rightarrow 6$ & 337396.6 & 0.18 & 0.04 & 3.6 & 0.67 & 0.54 & JCMT/Jan. 98 \\
\hline & $J=5 \rightarrow 4$ & 241016.2 & 0.27 & 0.11 & 3.6 & 1.06 & 0.19 & JCMT/Feb. 94 \\
\hline \multirow[t]{4}{*}{$\mathrm{CO}$} & $J=4 \rightarrow 3$ (a) $v_{\mathrm{lsr}}=-12.5 \mathrm{~km} \mathrm{~s}^{-1}$ & 461040.8 & $>29.0$ & 2.41 & 9.7 & 299.0 & 0.10 & JCMT/Jun. 94 \\
\hline & (b) $v_{\mathrm{lsr}}=-13.0 \mathrm{~km} \mathrm{~s}^{-1}$ & & 20.1 & 2.41 & 17.7 & 378.0 & 0.10 & JCMT/Jun. 94 \\
\hline & $J=3 \rightarrow 2$ (a) $v_{\mathrm{lsr}}=-90.7 \mathrm{~km} \mathrm{~s}^{-1}$ & 345796.0 & $>33.9$ & 0.57 & 12.7 & 457.0 & 0.14 & JCMT/Jun. 94 \\
\hline & (b) $v_{\mathrm{lsr}}=-89.6 \mathrm{~km} \mathrm{~s}^{-1}$ & & 27.7 & 0.57 & 19.1 & 563.0 & 0.14 & JCMT/Jun. 94 \\
\hline \multirow[t]{2}{*}{${ }^{13} \mathrm{CO}$} & $J=3 \rightarrow 2$ (a) $v_{\mathrm{lsr}}=-12.6 \mathrm{~km} \mathrm{~s}^{-1}$ & 330588.0 & $>17.3$ & 0.06 & 4.9 & 89.6 & 0.57 & JCMT/Jan. 98 \\
\hline & (b) $v_{\mathrm{lsr}}=-12.1 \mathrm{~km} \mathrm{~s}^{-1}$ & & 3.2 & 0.06 & 11.3 & 38.7 & 0.57 & JCMT/Jun. 98 \\
\hline \multirow[t]{2}{*}{$\mathrm{C}^{18} \mathrm{O}$} & $J=2 \rightarrow 1$ & 219560.3 & 7.85 & 0.34 & 2.8 & 23.7 & 0.21 & JCMT/Jun. 94 \\
\hline & $J=2 \rightarrow 1$ & 219560.3 & 8.81 & 0.48 & 2.8 & 26.6 & 0.11 & IRAM/Sep. 95 \\
\hline $\mathrm{C}^{17} \mathrm{O}$ & $J=3 \rightarrow 2$ & 337061.1 & 1.73 & 0.04 & 2.8 & 5.13 & 0.54 & JCMT/Jan. 98 \\
\hline $\mathrm{N}_{2} \mathrm{H}^{+}$ & $J=5 \rightarrow 4$ & 465824.8 & 0.25 & 0.16 & 2.6 & 0.7 & 0.40 & JCMT/Jan. 98 \\
\hline $\mathrm{NO}^{2} \Pi_{1 / 2}$ & $J=7 / 2 \rightarrow 5 / 2 F=4 \rightarrow 3,3 \rightarrow 2^{a}$ & 351051.7 & 0.061 & 0.056 & 6.7 & 0.4 & 1.07 & JCMT/Jun. 97 \\
\hline $\mathrm{NS}^{2} \Pi_{1 / 2}$ & $J=11 / 2 \rightarrow 9 / 2 F=6 \rightarrow 5,5 \rightarrow 4^{a}$ & 253970.7 & $\leq 0.08$ & 0.04 & - & - & 0.75 & JCMT/Jun. 97 \\
\hline \multirow[t]{5}{*}{ SO } & $N_{J}=9_{8} \rightarrow 8_{7}$ & 346528.6 & 0.50 & 0.04 & 4.7 & 2.50 & 0.55 & JCMT/Jan. 98 \\
\hline & $N_{J}=7_{6} \rightarrow 6_{5}$ & 261843.7 & 0.41 & 0.07 & 4.1 & 1.76 & 0.36 & JCMT/Jan. 98 \\
\hline & $N_{J}=6_{6} \rightarrow 5_{5}$ & 258255.8 & 0.18 & 0.07 & 4.7 & 0.90 & 0.73 & JCMT/Jun. 97 \\
\hline & $N_{J}=6_{5} \rightarrow 5_{4}$ & 219949.4 & 0.36 & 0.03 & 4.8 & 1.82 & 0.86 & JCMT/Jan. 98 \\
\hline & $N_{J}=6_{5} \rightarrow 5_{4}$ & 219949.4 & 0.63 & 0.03 & 3.2 & 2.18 & 0.86 & JCMT/Jun. 97 \\
\hline \multirow[t]{2}{*}{$\mathrm{SO}_{2}$} & $J_{K_{\mathrm{p}}, K_{\mathrm{o}}}=13_{2,12} \rightarrow 12_{1,11}$ & 345338.5 & 0.26 & 0.04 & 4.1 & 1.11 & 0.54 & JCMT/Jan. 98 \\
\hline & $J_{K_{\mathrm{p}}, K_{\mathrm{o}}}=8_{3,5} \rightarrow 8_{2,6}$ & 251210.6 & $\leq 0.80$ & 0.04 & - & - & 0.75 & JCMT/Jun. 97 \\
\hline \multirow[t]{3}{*}{$\mathrm{SiO}$} & $J=8 \rightarrow 7$ & 347330.6 & 0.17 & 0.05 & 4.3 & 0.77 & 0.54 & JCMT/Nov. 97 \\
\hline & $J=6 \rightarrow 5$ & 260518.0 & 0.06 & 0.05 & 16.1 & 0.95 & 0.36 & JCMT/Oct. 95 \\
\hline & $J=5 \rightarrow 4$ & 217104.9 & 0.10 & 0.03 & 5.5 & 0.59 & 0.86 & JCMT/Jun. 97 \\
\hline \multirow[t]{11}{*}{$\mathrm{CH}_{3} \mathrm{OH}$} & $J_{K}=5_{1} \rightarrow 4_{2} \mathrm{E}$ & 216945.6 & 0.05 & 0.03 & 6.38 & 0.32 & 0.86 & JCMT/Jun. 97 \\
\hline & $J_{K}=4_{2} \rightarrow 3_{1} \mathrm{E}$ & 218440.0 & 0.18 & 0.05 & 3.34 & 0.63 & 0.43 & JCMT/Oct. 95 \\
\hline & $J_{K}=2_{1} \rightarrow 1_{0} \mathrm{E}$ & 261805.7 & 0.09 & 0.07 & 4.88 & 0.48 & 0.36 & JCMT/Jan. 98 \\
\hline & $J_{K}=7_{-1} \rightarrow 6_{-1} \mathrm{E}$ & 338344.6 & 0.45 & 0.04 & 4.08 & 1.94 & 0.55 & JCMT/Jan. 98 \\
\hline & $J_{K}=7_{0} \rightarrow 6_{0} \mathrm{~A}^{+}$ & 338408.7 & 0.61 & 0.04 & 2.98 & 1.95 & 0.55 & JCMT/Jan. 98 \\
\hline & $J_{K}=7_{3} \rightarrow 6_{3} \mathrm{~A}^{-}$ & 338543.2 & 0.12 & 0.04 & 3.42 & 0.43 & 0.55 & JCMT/Jan. 98 \\
\hline & $J_{K}=7_{-3} \rightarrow 6_{-3} \mathrm{E}$ & 338559.9 & 0.14 & 0.04 & 2.15 & 0.06 & 0.55 & JCMT/Jan. 98 \\
\hline & $J_{K}=7_{1} \rightarrow 6_{1} \mathrm{E}$ & 338615.0 & 0.14 & 0.04 & 2.53 & 0.38 & 0.55 & JCMT/Jan. 98 \\
\hline & $J_{K}=7_{2} \rightarrow 6_{2} \mathrm{~A}^{+}$ & 338639.9 & 0.06 & 0.04 & 2.45 & 0.14 & 0.55 & JCMT/Jan. 98 \\
\hline & $J_{K}=7_{-2 / 2} \rightarrow 6_{-2 / 2} \mathrm{E}$ & 338722.0 & 0.21 & 0.04 & 4.05 & 0.92 & 0.55 & JCMT/Jan. 98 \\
\hline & $J_{K}=1_{1} \rightarrow 0_{0} \mathrm{~A}^{+}$ & 350905.1 & 0.28 & 0.06 & 2.80 & 0.84 & 1.07 & JCMT/Jun. 97 \\
\hline $\mathrm{HC}_{3} \mathrm{~N}$ & $J=24 \rightarrow 23$ & 218324.7 & 0.10 & 0.04 & 2.81 & 0.29 & 0.43 & JCMT/Oct. 95 \\
\hline \multirow[t]{2}{*}{$\mathrm{C}_{2} \mathrm{H}$} & $N_{J}=3_{4} \rightarrow 2_{3} \mathrm{~F}=4 \rightarrow 3,3 \rightarrow 2^{a}$ & 262004.3 & 1.02 & 0.07 & 5.08 & 5.50 & 0.36 & JCMT/Jan. 98 \\
\hline & $N_{J}=3_{3} \rightarrow 2_{2} \mathrm{~F}=3 \rightarrow 2,2 \rightarrow 1^{a}$ & 262067.5 & 0.69 & 0.07 & 5.76 & 4.22 & 0.36 & JCMT/Jan. 98 \\
\hline$\overline{\mathrm{HCN}}$ & $J=4 \rightarrow 3$ & 354505.5 & 2.23 & 0.23 & 4.03 & 9.55 & 0.26 & JCMT/May 96 \\
\hline $\mathrm{HC}^{15} \mathrm{~N}$ & $J=3 \rightarrow 2$ & 258157.0 & 0.08 & 0.07 & 6.77 & 0.60 & 0.73 & JCMT/Jun. 97 \\
\hline $\mathrm{H}^{13} \mathrm{CN}$ & $J=3 \rightarrow 2$ & 259011.8 & 0.23 & 0.07 & 4.27 & 1.06 & 0.36 & JCMT/Jan. 98 \\
\hline $\mathrm{DCN}$ & $J=3 \rightarrow 2$ & 217238.5 & 0.32 & 0.03 & 2.94 & 1.01 & 0.86 & JCMT/Jun. 97 \\
\hline
\end{tabular}

phase calibration and the data reduction, we used the Grenoble Software environment GAG.

We checked the total flux in the interferometric CS $J=$ $2 \rightarrow 1$ data and found that we lost $\approx 50-80 \%$ of the total flux in the velocity range $-14.5 \ldots-12 \mathrm{~km} \mathrm{~s}^{-1}$. Therefore, we added the IRAM 30-m CS $J=2 \rightarrow 1$ data (Fig. 1c) to the interferometer visibility data as zero-spacing information. The singledish weights were chosen in the order of the weights of the shortest interferometer baselines. The fine tuning of the singledish weights was performed such that the size of the resulting 
Table 2. continued.

\begin{tabular}{llcrrrrrr}
\hline \hline Molecule & transition & $\begin{array}{c}v \\
{[\mathrm{MHz}]}\end{array}$ & $\begin{array}{r}T_{\mathrm{mb}} \\
{[\mathrm{K}]}\end{array}$ & $\begin{array}{r}\mathrm{rms} \\
{[\mathrm{K}]}\end{array}$ & $\begin{array}{r}\Delta v \\
{\left[\mathrm{~km} \mathrm{~s}^{-1}\right]}\end{array}$ & $\begin{array}{c}\int T_{\mathrm{mb}} \mathrm{d} v \\
{\left[\mathrm{~K} \mathrm{~km} \mathrm{~s}^{-1}\right]}\end{array}$ & $\begin{array}{r}\text { spec. resol. } \\
{\left[\mathrm{km} \mathrm{s}^{-1}\right]}\end{array}$ & telescope/date \\
\hline $\mathrm{H}^{13} \mathrm{CO}^{+}$ & $J=4 \rightarrow 3$ & 346998.5 & 0.77 & 0.05 & 2.31 & 1.88 & 0.54 & JCMT/Nov. 97 \\
& $J=3 \rightarrow 2$ & 260255.5 & 0.92 & 0.05 & 2.47 & 2.40 & 0.36 & JCMT/Oct. 95 \\
$\mathrm{H}_{2} \mathrm{CO}$ & $J_{K_{\mathrm{p}}, K_{\mathrm{o}}}=7_{1,7} \rightarrow 6_{1,6}$ O & 491968.4 & 1.25 & 0.45 & 4.44 & 5.93 & 0.19 & JCMT/Jun. 94 \\
& $J_{K_{\mathrm{p}}, K_{\mathrm{o}}}=7_{1,7} \rightarrow 6_{1,6} \mathrm{O}$ & 491968.4 & 0.85 & 0.32 & 4.35 & 3.92 & 0.38 & JCM+T/Oct. 96 \\
& $J_{K_{\mathrm{p}}, K_{\mathrm{o}}}=5_{3,2} \rightarrow 4_{3,1} \mathrm{O}$ & 364288.9 & 0.29 & 0.13 & 2.99 & 0.94 & 0.26 & JCMT/Oct. 96 \\
& $J_{K_{\mathrm{p}}, K_{\mathrm{o}}}=5_{3,3} \rightarrow 4_{3,2} \mathrm{O}$ & 364275.2 & 0.46 & 0.13 & 2.00 & 0.99 & 0.26 & JCMT/Oct. 96 \\
& $J_{K_{\mathrm{p}}, K_{\mathrm{o}}}=5_{2,4} \rightarrow 4_{2,3} \mathrm{p}$ & 363945.9 & 0.37 & 0.13 & 4.72 & 1.87 & 0.26 & JCMT/Oct. 96 \\
& $J_{K_{\mathrm{p}}, K_{\mathrm{o}}}=3_{2,2} \rightarrow 2_{2,1} \mathrm{p}$ & 218475.6 & 0.35 & 0.04 & 2.66 & 1.00 & 0.43 & JCMT/Oct. 95 \\
& $J_{K_{\mathrm{p}}, K_{\mathrm{o}}}=3_{0,3} \rightarrow 2_{0,2} \mathrm{p}$ & 218222.2 & 2.18 & 0.04 & 2.68 & 6.21 & 0.43 & JCMT/Oct. 95 \\
$\mathrm{D}_{2} \mathrm{CO}$ & $J_{K_{\mathrm{p}}, K_{\mathrm{o}}}=6_{4,2} \rightarrow 5_{4,1}$ & 351492.0 & $0.32:$ & 0.22 & 2.76 & 0.93 & 0.26 & JCMT/May 96 \\
\hline $\mathrm{H}_{2} \mathrm{~S}$ & $J_{K_{\mathrm{p}}, K_{\mathrm{o}}}=2_{2,0} \rightarrow 2_{1,1}$ & 216710.4 & $\leq 0.06$ & 0.03 & - & - & 0.86 & JCMT/Jan. 98 \\
$\mathrm{HDO}$ & $J_{K_{\mathrm{p}}, K_{\mathrm{o}}}=1_{0,1} \rightarrow 0_{0,0}$ & 464924.5 & $\leq 1.1$ & 0.54 & - & - & 0.40 & JCMT/Dec. 96 \\
{$[\mathrm{C} \mathrm{I}]$} & ${ }^{3} \mathrm{P}_{1} \rightarrow{ }^{3} \mathrm{P}_{0}$ & 492160.7 & 9.08 & 0.45 & 4.2 & 40.7 & 0.16 & JCMT/Jun. 94 \\
\hline
\end{tabular}

${ }^{a}$ Blend of two hyperfine components.

synthesized beam (clean beam) is very close to the size without zero-spacing correction. In the continuum, all of the single-dish flux was recovered by the interferometer, due to the compact source size.

Maps of $256 \times 256$ square pixels with $0.5^{\prime \prime}$ pixel size were produced by Fourier transforming the calibrated visibilities, using natural weighting. The synthesized beam sizes (HPBW) are $2.72^{\prime \prime} \times 2.21^{\prime \prime}(=2720 \mathrm{AU} \times 2210 \mathrm{AU})$ for the continuum data and $2.73^{\prime \prime} \times 2.22^{\prime \prime}$ for the line map (with zero-spacing correction), each with a position angle of $15^{\circ}$.

The initial CS maps showed a strong $(0.11 \mathrm{Jy})$ peak at phase center, which was independent of velocity. Since the peak is also seen in the continuum maps, the continuum data were subtracted from the original line (+continuum) data in the uv plane. The resulting CS line flux at the position of the object, summed over all channels, decreased by a factor of 1.8 to $1.248 \mathrm{Jy} \mathrm{km} \mathrm{s}^{-1} /$ beam.

\subsection{Observations and results with ISOPHOT}

Observations of AFGL 490 were carried out using the spectrophotometer (PHT-S), the photopolarimeter (PHT-P) and the photometric camera (PHT-C200) of the imaging photo-polarimeter ISOPHOT aboard ISO (Infrared Space Observatory). The spectrophotometer subsystem consists of two low-resolution grating spectrometers covering the wavelength ranges 2.5-5 $\mu \mathrm{m}$ (PHT-SS) and 5.8-11.6 $\mu \mathrm{m}$ (PHT-SL). A detailed description of ISOPHOT is given by Lemke et al. (1996). The multi-aperture filter photometry has been performed with the detector PHT-P1 of the photopolarimeter to obtain flux values for the wavelengths $\lambda=3.6,4.85,7.3,7.7$, $10.0,12.8$, and $16.0 \mu \mathrm{m}$ applying an aperture of $23^{\prime \prime}$. For $7.3 \mu \mathrm{m}$, additional measurements with apertures of $52^{\prime \prime}$ and $79^{\prime \prime}$ were performed. The detector PHT-P2 was used to obtain aperture filter photometry for $\lambda=20.0$ and $25.0 \mu \mathrm{m}$, and with PHT-P3, we got $5 \times 5$ raster point measurements for $\lambda=60$ and $100 \mu \mathrm{m}$. With the photometric camera PHT-C200, we did multi-filter mapping (raster $4 \times 2$ ) at $\lambda=120$ and $200 \mu \mathrm{m}$.
The ISO photometry observations were done on August 28, 1997, and the spectrophotometer subsystem was used on August 25, 1996.

The raw data were reduced using the standard procedures of the PHT Interactive Analysis (PIA) software (version 7.3) ${ }^{3}$. The absolute photometric accuracy of the data is better than $\pm 30 \%$.

The results of the ISOPHOT photometry are compiled in Table 3. The flux densities and their errors were determined with and without a colour correction (see for details: PIA software manual). In the case of the colour correction, we applied a black body model of different temperatures $T_{\mathrm{bb}}$. Black bodies with temperatures $T_{\mathrm{bb}} \geq 400 \mathrm{~K}$ show no large differences in the resulting flux densities.

\subsection{Observations with ISO-SWS}

Complementary spectral information has been obtained with the ISO Short Wavelength Spectrometer (SWS) at infrared wavelengths. AFGL 490 was observed on March 27, 1998 (revolution 863), using the high-resolution SWS06 grating mode centered at $\mathrm{RA}(1950)=03^{\mathrm{h}} 23^{\mathrm{m}} 38.95^{\mathrm{s}}, \operatorname{Dec}(1950)=$ $+58^{\circ} 36^{\prime} 33^{\prime \prime}$, with a beam size of $\sim 14^{\prime \prime} \times 20^{\prime \prime}$ between 4.0 and $9.0 \mu \mathrm{m}$ and $\sim 14^{\prime \prime} \times 27^{\prime \prime}$ between 12.0 and $16.5 \mu \mathrm{m}$. The data have been reduced using standard routines within the SWS Interactive Analysis package (version 10). The final spectra have been rebinned to a spectral resolving power of $\sim 2600$ between 4.0 and $5.3 \mu \mathrm{m}, \sim 1500$ between 5.3 and $9.0 \mu \mathrm{m}$ and $\sim 2000$ between 12.0 and $16.5 \mu \mathrm{m}$. Also, a low-resolution $(\lambda / \Delta \lambda \sim 900)$ SWS01 speed 3 spectrum between $\lambda=2.5$ and $45 \mu \mathrm{m}$ was observed on August 17, 1997 (revolution 640) toward the position $\operatorname{RA}(1950)=03^{\mathrm{h}} 23^{\mathrm{m}} 39.15^{\mathrm{s}}$, Dec $(1950)=$ $+58^{\circ} 36^{\prime} 36^{\prime \prime}$. The results are discussed in Sect. 7.3.

\footnotetext{
${ }^{3}$ PIA is a joint development by the ESA Astrophysics Division and the ISOPHOT consortium led by the Max Planck Institute for Astronomy (MPIA), Heidelberg.
} 
Table 3. Results of the multi-aperture photometry with ISOPHOT. The flux density values and their errors were determined without and with a colour correction using a black-body model with two different temperatures $T_{\mathrm{bb}}$.

\begin{tabular}{ccccc}
\hline \hline & & \multicolumn{3}{c}{ Colour correction } \\
\cline { 3 - 5 }$\lambda$ & aperture & no corr. & $T_{\mathrm{bb}}=400 \mathrm{~K}^{a}$ & $T_{\mathrm{bb}}=1000 \mathrm{~K}$ \\
{$[\mu \mathrm{m}]$} & {$\left[{ }^{\prime \prime}\right]$} & $S_{v}[\mathrm{Jy}]$ & $S_{v}[\mathrm{Jy}]$ & $S_{v}[\mathrm{Jy}]$ \\
\hline 3.60 & $23^{\prime \prime}$ & $11.3( \pm 0.03)$ & $10.9( \pm 0.03)$ & $11.3( \pm 0.03)$ \\
4.80 & $23^{\prime \prime}$ & $26.3( \pm 0.06)$ & $26.0( \pm 0.06)$ & $26.5( \pm 0.07)$ \\
7.30 & $23^{\prime \prime}$ & $37.9( \pm 0.1)$ & $39.9( \pm 0.1)$ & $36.4( \pm 0.1)$ \\
7.30 & $52^{\prime \prime}$ & $43.7( \pm 0.1)$ & $46.0( \pm 0.1)$ & $42.1( \pm 0.1)$ \\
7.30 & $72^{\prime \prime}$ & $44.4( \pm 0.1)$ & $46.8( \pm 0.1)$ & $42.7( \pm 0.1)$ \\
7.70 & $23^{\prime \prime}$ & $63.6( \pm 0.5)$ & $64.2( \pm 0.5)$ & $63.0( \pm 0.5)$ \\
10.00 & $23^{\prime \prime}$ & $36.8( \pm 0.1)$ & $37.1( \pm 0.1)$ & $36.4( \pm 0.1)$ \\
12.80 & $23^{\prime \prime}$ & $92.4( \pm 0.2)$ & $92.4( \pm 0.2)$ & $91.4( \pm 0.2)$ \\
15.00 & $23^{\prime \prime}$ & $127.0( \pm 1.1)$ & $128.0( \pm 1.1)$ & $128.0( \pm 1.1)$ \\
20.00 & $23^{\prime \prime}$ & $207.0( \pm 0.6)$ & $213.0( \pm 0.6)$ & $207.0( \pm 0.5)$ \\
25.00 & $23^{\prime \prime}$ & $266.0( \pm 0.5)$ & $228.0( \pm 0.5)$ & $213.0( \pm 0.5)$ \\
25.00 & $52^{\prime \prime}$ & $283.0( \pm 0.5)$ & $241.0( \pm 0.5)$ & $226.0( \pm 0.5)$ \\
25.00 & $72^{\prime \prime}$ & $303.0( \pm 0.4)$ & $259.0( \pm 0.4)$ & $242.0( \pm 0.4)$ \\
60.00 & $43.5^{\prime \prime}$ & $334.0( \pm 1.4)$ & $334.6( \pm 1.4)$ & $335.0( \pm 1.4)$ \\
100.00 & $43.5^{\prime \prime}$ & $574.0( \pm 1.8)$ & $574.4( \pm 1.8)$ & $573.0( \pm 1.7)$ \\
120.00 & $89.4^{\prime \prime}$ & $854.0( \pm 11)$ & $712.0( \pm 11)$ & $706.0( \pm 1.1)$ \\
200.00 & $89.4^{\prime \prime}$ & $641.0( \pm 7.0)$ & $622.0( \pm 7.0)$ & $622.0( \pm 7.0)$ \\
\hline & & & &
\end{tabular}

${ }^{a}$ Value used for the models in Sect. 6.

\section{Structure of the envelope}

Figure 1 summarizes the mapping results of the integrated intensities for different line transitions. For the sake of comparison, all maps were plotted for the same sky area. The central map position refers to $\mathrm{RA}(1950)=03^{\mathrm{h}} 23^{\mathrm{m}} 39.0^{\mathrm{s}}, \operatorname{Dec}(1950)=$ $58^{\circ} 36^{\prime} 33^{\prime \prime}$. 0 in all individual figures, close to the near-infrared position.

The general morphology of the maps is in accordance with earlier line measurements (e.g. Mitchell et al. 1992, 1995; van der Tak et al. 2000b). Whereas the CS maps show a spherically symmetric structure, all $\mathrm{C}^{18} \mathrm{O} J=2 \rightarrow 1$ maps reveal a more elongated structure in the north-south direction similar to that found in HCN $J=4 \rightarrow 3$ by Kawabe et al. (1987) as well as in the $\lambda 0.87 \mathrm{~mm}$ and $\lambda 1.3 \mathrm{~mm}$ dust continuum emission by Chini et al. (1991). This structure is neither perpendicular to the high-velocity outflow direction (northeastsouthwest) nor to the proposed disk orientation (northwestsoutheast), however, it fits the location of the scattered light in the $K$-band image obtained by Hodapp (1994). The higher excitation lines are slightly more extended than the beam sizes, suggesting a power-law structure of the envelope with a central concentration.

We studied the line wings in the lower-excitation CS transitions because these data cover a larger part of the envelope compared with our CO data. Our maps of red and blueshifted CS $J=2 \rightarrow 1$ line wing emission are shown in Fig. 2. The result implies a rather complicated kinematical situation.
The global structure resembles the CS measurements of Kawabe et al. (1984). Their blue- and red-shifted CS emission is shown in Fig. 2b as two lobes (blue: southwest - red: northeast). These lobes have the same orientation as the proposed disk-like structure seen by Mundy \& Adelmann (1988) and are perpendicular to the high-velocity large-scale CO outflow (thin line lobes in Fig. 2b: direction northeast-southwest). Kawabe et al. (1984) discussed a model similar to that of Orion IRc2 where a dense, expanding gas torus/disk surrounds the central energy source and is located perpendicular to the large-scale outflow direction.

However, Nakamura et al. (1991) concluded from modelling of their interferometer CS $J=2 \rightarrow 1$ data an opposite location of red- and blue-shifted gas possibly associated with a disk. They interpreted these data as infalling gas. We should stress that the global structure of our single-dish CS line wings could be produced by different clumps or due to absorption because the source AFGL 490 is not located in the centre of the red- and blue-shifted line emission. The density of the gas in the high-velocity large-scale outflow found in $\mathrm{CO}$ (see, e.g., Lada \& Harvey 1981) is possibly too low to be traced with our CS measurements.

Integrating the $\mathrm{C}^{18} \mathrm{O}$ emission inside a radius of $20^{\prime \prime}$, which coincides with the inner envelope, we can estimate a mean $\mathrm{H}_{2}$ column density of $6 \ldots 8 \times 10^{22} \mathrm{~cm}^{-2}$ applying the conversion $N\left(\mathrm{H}_{2}\right)\left[\mathrm{cm}^{-2}\right]=3 \times 10^{21} \int T_{\mathrm{mb}} \Delta v$ (Frerking et al. 1982). Assuming spherical symmetry, we obtain an envelope mass of 40-50 $M_{\odot}-$ a value which agrees with earlier estimates for the inner envelope (e.g. Kawabe et al. 1984).

\section{Inner envelope and disk}

\subsection{Inner envelope}

Line measurements: The total integrated line intensity interferometer map of CS $J=2 \rightarrow 1$ (continuum subtracted, zerospacing corrected) and the continuum emission at $97.98 \mathrm{GHz}$ obtained with the $\mathrm{PdB}$ interferometer are compared to the $2.2 \mu \mathrm{m}$ map by Hodapp (1994) in Fig. 3. Our CS line map (Fig. 3b) shows an extended dense gas structure like a bar with a size of $22000 \mathrm{AU} \times 6000 \mathrm{AU}$ and a position angle of $-40^{\circ}\left( \pm 5^{\circ}\right)$. The overall structure of this bar agrees with the morphology found in the lower resolution CS measurements by Nakamura et al. $(1991: 30000 \times 15000-20000 \mathrm{AU})$ and in the ${ }^{13} \mathrm{CO}$ data by Mundy \& Adelman (1988: $45000 \times$ $14000 \mathrm{AU})$. In addition, the CS map suggests that this elongated bar-like structure consists of different individual intensity peaks. Comparison of the CS map with the near-infrared image by Hodapp (1994) shows that the shape of the scattered $2.2 \mu \mathrm{m}$ radiation fits well the location of the gaseous envelope around AFGL 490 (Fig. 3a).

Spectra extracted from the interferometer data show that the CS emission in the velocity range $-14.5 \ldots-12 \mathrm{~km} \mathrm{~s}^{-1}$ is strongly (self-)absorbed. Various examples of spectra taken at different map positions are shown in Fig. 3d, both before and after zero-spacing correction. Only the densest gas is present in the data without zero-spacing correction. Whereas spectra of the outer region around AFGL 490 (e.g. (7) and (9) of 


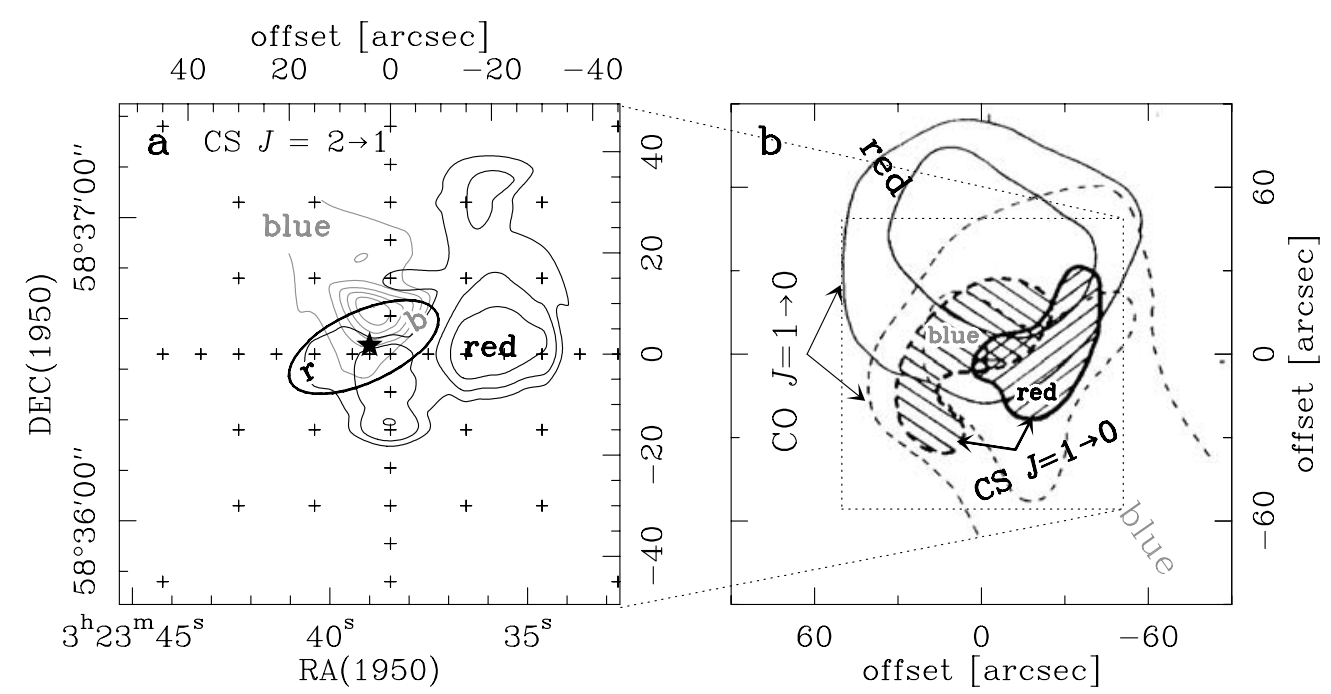

Fig. 2. a) Map of the integrated line wing emission of CS $J=2 \rightarrow 1$ (blue: velocity range of $-20 \mathrm{~km} \mathrm{~s}^{-1} \leq v_{\mathrm{lsr}} \leq-15 \mathrm{~km} \mathrm{~s}^{-1}$; red: $-11 \mathrm{~km} \mathrm{~s} \mathrm{~s}^{-1} \leq$ $\left.v_{\mathrm{lsr}} \leq-6 \mathrm{~km} \mathrm{~s}^{-1}\right)$. Contour levels are $70 \%$ to $90 \%$ of the red peak emission $\left(1.2 \mathrm{~K} \mathrm{~km} \mathrm{~s}^{-1}\right)$ and $50 \%$ to $90 \%$ of the blue peak emission ( $3.0 \mathrm{~K} \mathrm{~km} \mathrm{~s}^{-1}$ ). The thick ellipse shows the extent of the CS $J=2 \rightarrow 1$ emission measured by Nakamura et al. (1991). The red- and blue-shifted parts are indicated with $r$ and b. b) Comparison of our mapped area with previous results obtained by Kawabe et al. (1984).

Fig. 3) show a narrow Gaussian line profile centered at $v_{\mathrm{lsr}}=$ $-13.17 \mathrm{~km} \mathrm{~s}^{-1}$, the shape of the spectra of the bar-like region clearly indicates absorption features. It is very unlikely that there are two (or more) velocity components well separated at $v_{\text {lsr }}=-13.17 \mathrm{~km} \mathrm{~s}^{-1}$ (see Fig. $3 \mathrm{~d}$ ). Therefore, we conclude that the CS $J=2 \rightarrow 1$ emission is highly optically thick towards AFGL 490.

Due to the strong absorption, the interpretation of the general gas dynamics is complicated. The bar-like structure in CS, indicating "single dense clumps", may be partly an artificial structure created by the remaining non-absorbed line profiles. We should note that, at the position of AFGL 490, the total integrated intensity of CS is lower than in the gas clumps located to the southeastern and northwestern side of AFGL 490. This is probably caused by a stronger absorption towards the source compared to the surrounding region. Based on the $3 \mathrm{~mm}$ emission at this position, it can be excluded that there is a real "hole" of material.

For comparison with the Nakamura et al. (1991) data, we created position-velocity cuts (see Fig. 6) along the bar-like structure and found a very similar position-velocity diagram. Whereas Nakamura et al. fitted their position-velocity diagram with infalling gas of constant velocity and rigid rotation in an inclined disk, we conclude that the position-velocity structure, using the strongly absorbed CS emission, does not trace the real gas motion in the velocity range $v_{\mathrm{lsr}}=-14.5 \ldots-12 \mathrm{~km} \mathrm{~s}^{-1}$. In addition, the extent of the dense bar-like structure is rather large compared with other disk-like structures around intermediatemass pre-main sequence stars, like, e.g. Herbig Ae stars (Mannings \& Sargent 1997, 2000) with sizes of a few hundred AU and gas in Keplerian rotation. Therefore, we suggest that the remaining CS $J=2 \rightarrow 1$ line profiles indicate more the existence of a very dense and elongated envelope around AFGL 490, perhaps the remnant of a flattened cloud core, and not a real (Keplerian) disk with such a large extension. Interesting to note is the central "channel" perpendicular to the densest bar-like structure at the position of AFGL 490 (Fig. 4), which corresponds well with the direction of the largescale high-velocity outflow.

Continuum measurements: In contrast to the more extended material seen in CS, the continuum map (Fig. 3c) shows a point source at the position of AFGL 490 with only a slight extension at the $3 \sigma$ level. The more intense CS clumps located to the southeast and northwest side of AFGL 490, have no counterpart in the continuum emission.

The coordinates of the $3 \mathrm{~mm}$ peak emission are $\mathrm{RA}(2000)=$ $03^{\mathrm{h}} 27^{\mathrm{m}} 38.81( \pm 0.26)^{\mathrm{s}}$ and $\operatorname{Dec}(2000)=+58^{\circ} 47^{\prime} 00.28( \pm 0.2)^{\prime \prime}$ which coincide well with the peak positions given by Nakamura et al. (1991) and Campbell et al. (1986, $2 \mathrm{~cm}$ VLA measurements). The peak flux amounts to $0.11 \mathrm{Jy} /$ beam. Above the $3 \sigma$ level, a flux of $0.24 \mathrm{Jy}$ was detected which agrees very well with the results obtained by Nakamura et al. (1991) and Mundy \& Adelman (1988). Based on the flux measurements for wavelengths larger than $1 \mathrm{~cm}$ by Campbell et al. (1986) and Simon et al. (1983), we estimate that the contribution of free-free radiation to the total flux at $97.98 \mathrm{GHz}$ is $\approx 17( \pm 3)$ mJy only, which is less than $10 \%$ of the total flux.

The continuum source can be described by a 2-dimensional Gaussian with full axes at half maximum of $3.2^{\prime \prime} \times 2.25^{\prime \prime}$ at a position angle of $10^{\circ}$. The deconvolved source size is $1.7^{\prime \prime} \times$ $0.38^{\prime \prime}$ at a position angle of $5.4^{\circ}$ which corresponds to a continuum source size of only $1700 \mathrm{AU} \times 380 \mathrm{AU}$. This value is smaller than the result found by Mundy \& Adelman (1988) of $2600 \times 1500 \mathrm{AU}$. However, the major axis of our continuum source is located perpendicular to the structure found by Mundy \& Adelman and located in the direction of the largescale outflow. Here we must stress that the dimension of the millimetre source is only slightly larger than the actual beam size and we will not base any further discussion on the "shape" of the object. 


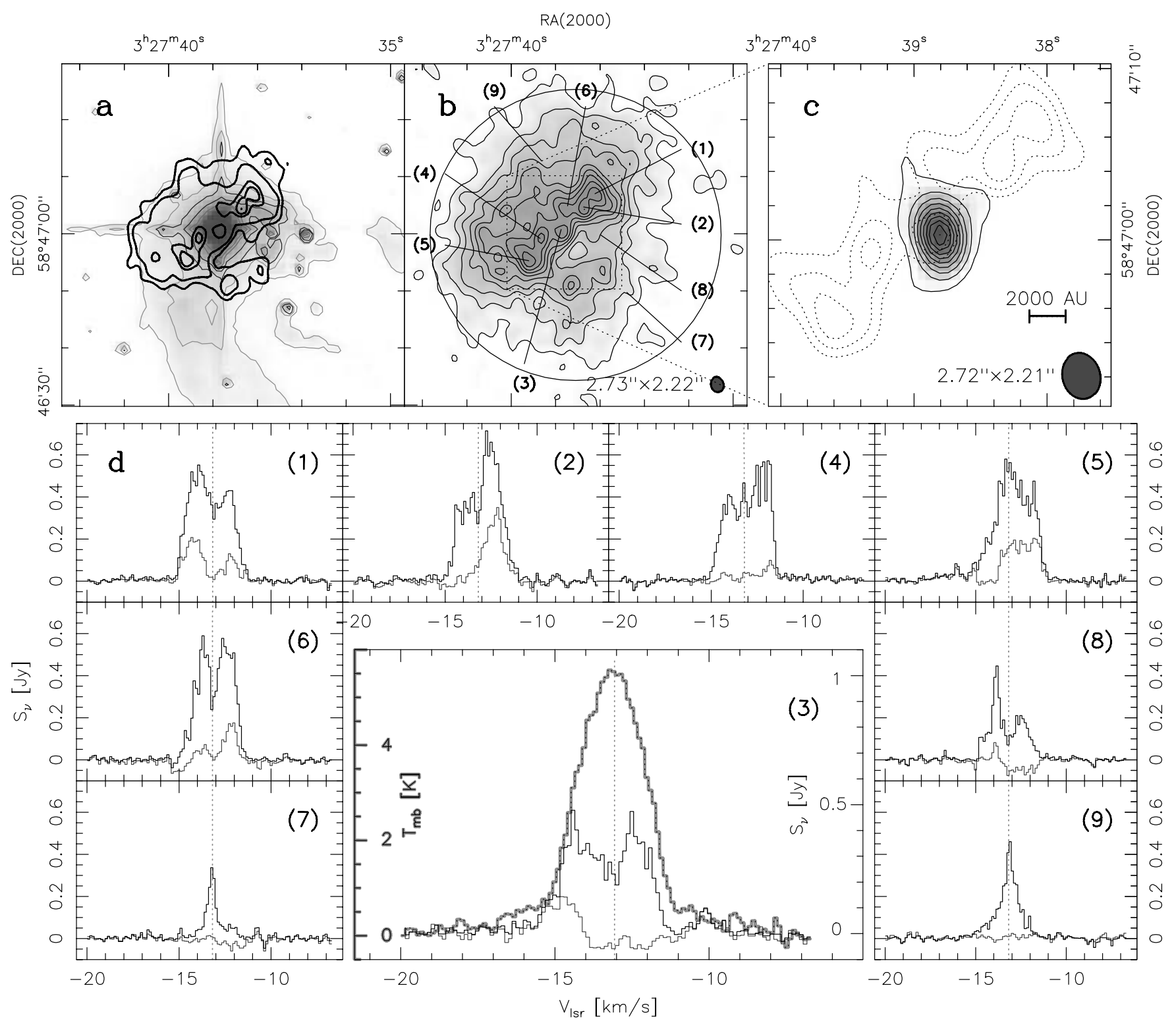

Fig. 3. a) Integrated CS $J=2 \rightarrow 1$ line emission map (continuum subtracted and zero-spacing corrected) measured with the PdB interferometer (thick contour lines) is overlaid with the $K$-band image obtained by Hodapp (1994). Contour lines are the same as in Fig. b) $70 \%, 80 \%, \&$ $90 \%$ of the emission peak. b) Same as panel a, but with contour levels at 10 to $90 \%$ of the peak emission $\left(=1.42 \mathrm{Jy} \mathrm{beam}^{-1} \mathrm{~km} \mathrm{~s}^{-1}\right)$, where the $10 \%$ contour line corresponds to the $3 \sigma$ noise level. The clean beam is given with a dark filled ellipse and the primary beam of $51^{\prime \prime}$ is indicated by the large circle. The numbers in brackets correspond to the spectra shown in panel d). c) Continuum map (grey scale image + solid contours, details see Fig. 5) at $97.98 \mathrm{GHz}$ obtained with PdB. The contour lines of 70\%, 80\%, \& $90 \%$ of the CS emission are superimposed as dotted lines. d) Examples of spectra extracted from different positions of the CS line map in panel b. Spectra in black represent interferometer data with zero-spacing correction. Grey spectra indicate data without zero-spacing. At the position (3) which is the position of AFGL 490, we overlaid the IRAM $30 \mathrm{~m}$ spectra (thick, grey) of the single-dish map position $[0,0]$. The dotted line in all spectra indicates the velocity at $v_{\mathrm{lsr}}=$ $-13.17 \mathrm{~km} \mathrm{~s}^{-1}$.

\subsection{A disk around AFGL 490 ?}

The spectra in Fig. 4a show red and blue line wing emission well separated from the remaining main line profile. Maps of the integrated red and blue line wing emission indicate different flow systems related to YSOs around AFGL 490 (see Fig. 4b), which will be discussed in Sect. 5.4.

One "blue-red" system is centered at the position of AFGL 490. The location of the red and the blue emission would strongly speak in favour of a disk because the orientation is perpendicular to the large-scale outflow of AFGL 490. However, these data alone do not allow to decide if the gas represents indeed a disk or if this is an outflow produced by a very nearby low-mass companion. Figure 5 shows two overlays of the $3 \mathrm{~mm} \mathrm{PdB}$ continuum measurements and the blue- and red-shifted CS line wing emission with both the $2 \mathrm{~cm}$ VLA emission obtained by Campbell et al. (1986) and the high-resolution speckle $H$-band image published by Hoare et al. (1996). The $H$-band image shows no other point source in the immediate vicinity of AFGL 490 favouring the disk 

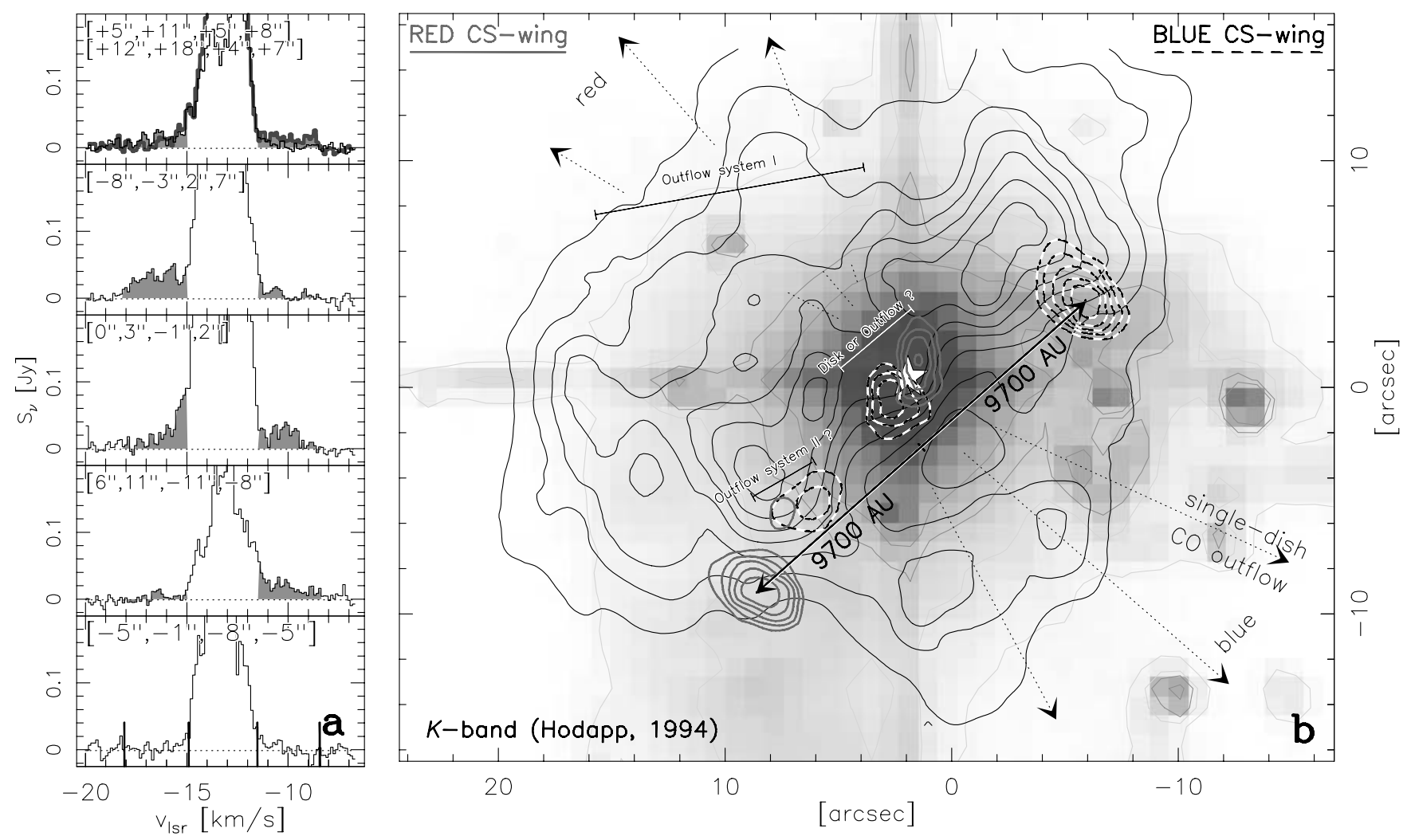

Fig. 4. a) Spectra extracted from the PdB images in the offset ranges $\left[x_{1}, x_{2}, y_{1}, y_{2}\right]$. b) $K$-band image obtained by Hodapp (1994) overlaid with the CS $J=2 \rightarrow 1$ emission (thin contour lines, the same as Fig. $3 \mathrm{~b}$ ), the blue $\left(-18.2 \leq v_{\mathrm{lsr}} \leq-15.0 \mathrm{~km} \mathrm{~s}^{-1}\right)$ and the red $\left(-11.5 \leq v_{\mathrm{lsr}} \leq\right.$ $-8.35 \mathrm{~km} \mathrm{~s}^{-1}$ ) CS line wing emission. The position of the continuum source is marked by a star. The direction of the large-scale high-velocity single-dish CO outflow (Lada \& Harvey 1981) is indicated.

interpretation. In addition, the dynamical age of a small-scale outflow would be of the order of $0.2-6 \times 10^{3}$ yrs (inclination angle between $10^{\circ}$ and $80^{\circ}$ ), which is very small compared to the dynamical timescale of the large-scale high-velocity outflow of $1.8 \times 10^{4}$ yrs (Churchwell 1999), which is associated with AFGL 490.

Most of the extended near-infrared emission, extended in the NW-SE direction (see Fig. 5), is interpreted as scattered light. The size and the orientation of this elongated nebulosity is similar to the extended $2 \mathrm{~cm}$ VLA emission observed by Campbell et al. (1986). It is remarkable that the more recent speckle NIR images and VLA data, obtained by Hoare (2001), show no extended emission at these wavelengths. This may be due to variability of the source.

We should note that we shifted the $2 \mathrm{~cm}$ VLA image by $0.3^{\prime \prime}$ to bring the peak positions of the VLA and the $3 \mathrm{~mm}$ continuum image in agreement (uncertainties: PdB 0.2", VLA $\left.0.1^{\prime \prime}\right)$. In this way, the peaks of the blue- and the redshifted line wing emission are located at the boundaries of both the $2 \mathrm{~cm}$ VLA emission as well as the $H$-band image. For comparison, the typical size of the more evolved disks around Herbig Ae stars (Mannings \& Sargent 1997, 2000, radius of $400 \mathrm{AU}$ ) is indicated.

Further evidence for the existence of a disk comes from the distribution of the high-velocity gas. Two position-velocity diagrams are plotted in Fig. 6. Cut $\mathrm{A}-\mathrm{A}^{\prime}$ crosses the position of AFGL 490 and B-B' cuts the two gas blobs of a possible jet.
Based on the strong absorption in the central CS line, the inner part of the diagrams is blocked out.

In the case of the red and blue line emission around AFGL 490 (Cut A-A', Fig. 6a), the velocity field of the wing emission is compared with a simple model of Keplerian motion with a mass distribution introduced by Vogel et al. (1985). In the rotational equilibrium model, a central star and a disk mass linearly increasing with radius are assumed. A central mass of $M_{\star}=8( \pm 1) M_{\odot}$ is used in agreement with the mass of a mainsequence star of spectral type B2...B3 and a "disk" radius of $4 "$ (=4000 AU), which is rather large compared with the more evolved disks of Herbig Ae stars. However, large gas tori of comparable extent were found around another embedded B star (G 192.16-3.82; Shepherd \& Kurtz 1999; Shepherd et al. 2001) and the (more massive) object IRc2(I) in the Orion BN-KL region (Plambeck et al. 1982; Gezari et al. 1998; Greenhill et al. 1998; Schreyer et al. 1999).

For the mass of the model, we took values found for the region of the continuum source (see Sect. 5.3 and Table 4), where a disk mass between $7 . . .9 M_{\odot}$ gives the best fit. We used different inclination angles, where the best fits lie between $i=$ $17 \ldots 23^{\circ}$. The calculated curve for a disk mass of $8 M_{\odot}$, centered at $v_{\text {lsr }}=-13.2 \mathrm{~km} \mathrm{~s}^{-1}$, and an inclination angle of $20^{\circ}$ is overplotted in Fig. 6a as thick dashed line. The curve fits reasonably well the outer parts of the position-velocity diagram with the exception of the emission very close to the centre. Because of the missing kinematic information from the inner region 


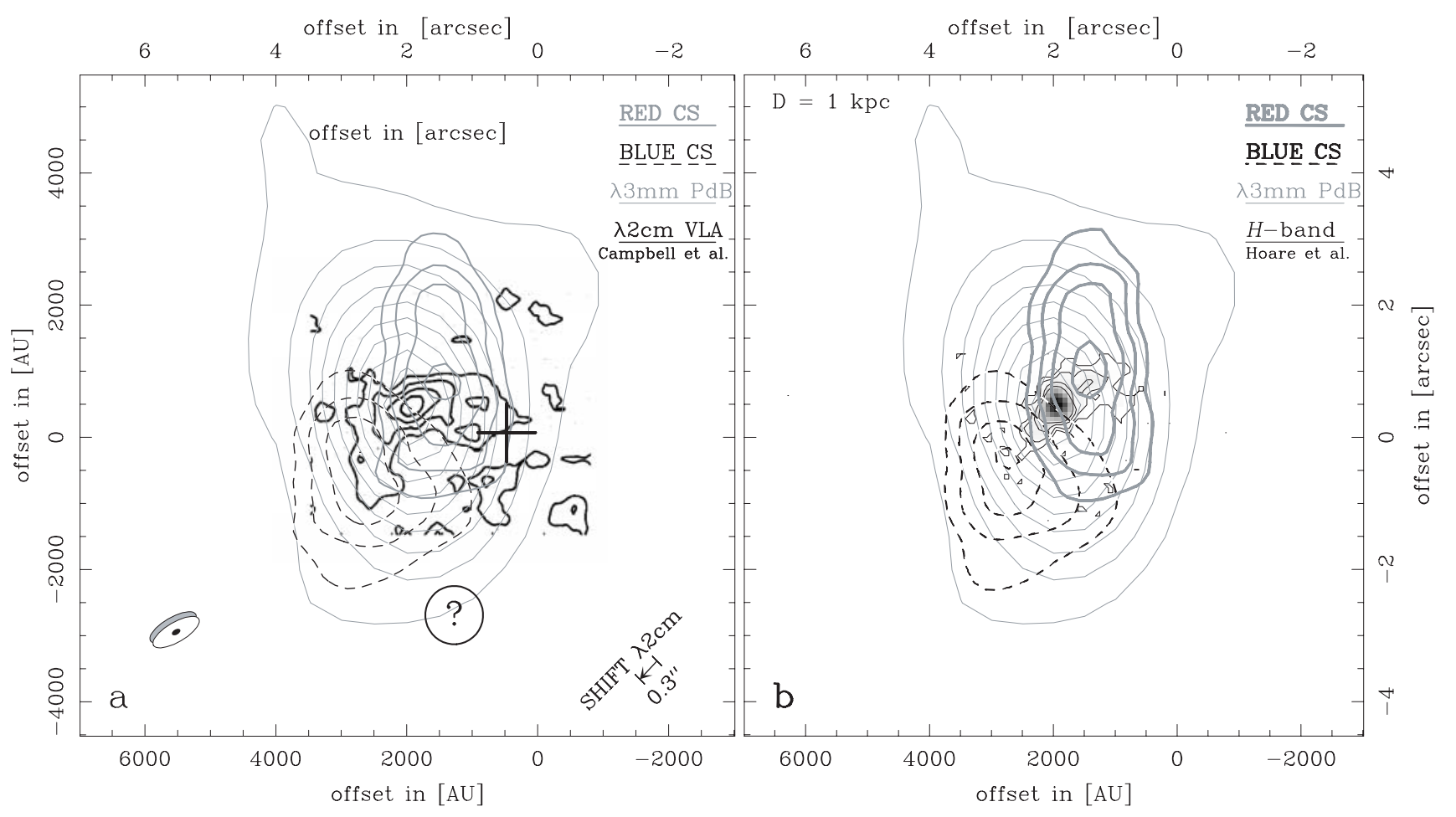

Fig. 5. Overlay of our $3 \mathrm{~mm}$ continuum map (contour levels $10 \%$ to $90 \%$ of the peak $=0.11 \mathrm{Jy} / \mathrm{beam}$, where the $10 \%$ level corresponds to $4 \sigma$ ) and the red- and the blue-shifted CS line wing emission (same contours as in Fig. 4) with a) the 2 cm continuum VLA map obtained by Campbell et al. (1986) which is shifted by $0.3^{\prime \prime}$ to the peak of the $3 \mathrm{~mm}$ continuum map. The cross marks the near-infrared source position. The circle with the question mark indicates the possible position of the low-mass star probably causing the red and the blue gas blobs at a distance of $9700 \mathrm{AU}$ (see Fig. 4). The schematic disk at the bottom left shows the extent of a typical disk around the more evolved Herbig Ae stars with a radius of 400 AU (Mannings \& Sargent 1997, 2000). b) Overlay with the speckle $H$-band image obtained by Hoare et al. (1996).

(see Fig. 6), the inclination angle could possibly be smaller, but not larger.

The separation between the red- and blueshifted peaks of $\approx 1800 \mathrm{AU}$ is larger than radiative transfer models predict for the destruction radius of the dust grains (Sect. 6): 10-15 AU for a star with $T_{\star}=2 \times 10^{4} \mathrm{~K}$. An explanation could be that the inner zone without gas and dust around the star is due to the presence of an accelerating stellar wind (Bunn et al. 1995), Simon et al. (1983) estimated a maximum extent of the ionized region of $\leq 100 \mathrm{AU}$. An assumed ionized region of 50...100 AU would match well the proposal that a larger inner wind zone is surrounded by a gaseous disk.

We also checked the visibilities of the continuum measurements to find out if they are dominated by an unresolved (pointlike) component or by the emission of an envelope. The results of our investigation are summarized in Table 5 and shown in Fig. 7a-d. In general, a point(-like) source in the centre and any envelope structure around the point source fit the observed data well. The point source can be a "real" point source or a very narrow $\left(r_{\text {out }}<0.25^{\prime \prime}\right)$ ring, a Gaussian, or a power-law model where the brightness falls off as $\sim r^{-2}$ or $\sim r^{-3}$. The envelope can be a Gaussian or a ring, but this is of minor importance for the quality of the fit.

In all cases, the derived flux density of the point(-like) source is $140( \pm 20) \mathrm{mJy}$. For a Gaussian-type shell, the flux is $80( \pm 9) \mathrm{mJy}$, while for an annulus, it is only $38( \pm 4) \mathrm{mJy}$.
The outer radius (HWHM) of the Gaussian shell is about $3.5( \pm 0.5)^{\prime \prime}$. The annulus would be a rather broad ring with $r_{\text {in }} \sim$ $0.5^{\prime \prime}$ and $r_{\mathrm{out}}=4 \ldots 5^{\prime \prime}$.

In Sect. 6, we develop a quantitative model of the envelope based on radiative transfer calculations. The best-fitting model with a single density gradient of $r^{-1}$ was used to calculate the visibility for the envelope contribution (see Fig. 7f). Also in this case, the $u v$ data are clearly dominated by a point source, very much favouring a disk interpretation. The comparison of the observed and the calculated visibilities indicates that the observed flux at $3 \mathrm{~mm}$ mainly comes from a central source with a radius smaller than $0.6^{\prime \prime}$ (=600 AU). Model calculations demonstrate that different density gradients in the envelope have no influence on the shape of the resulting visibilities. This is easy to understand because the contribution from the envelope is practically below the noise level of the measurements.

All results from both the line observations and the continuum point to the presence of a disk around AFGL 490 which has a radius smaller than about 500 AU. The disk mass, based on the Keplerian model (however with an outer radius of $4000 \mathrm{AU})$, is of the order of the mass of the central star. The result $M_{\text {disk }} \approx M_{\text {star }}$ agrees very well with the findings by Shepherd et al. (2001) and is 10 to 100 times that typically found for disks around T Tauri stars and Herbig Ae stars (Mannings \& Sargent 1997, 2000). 


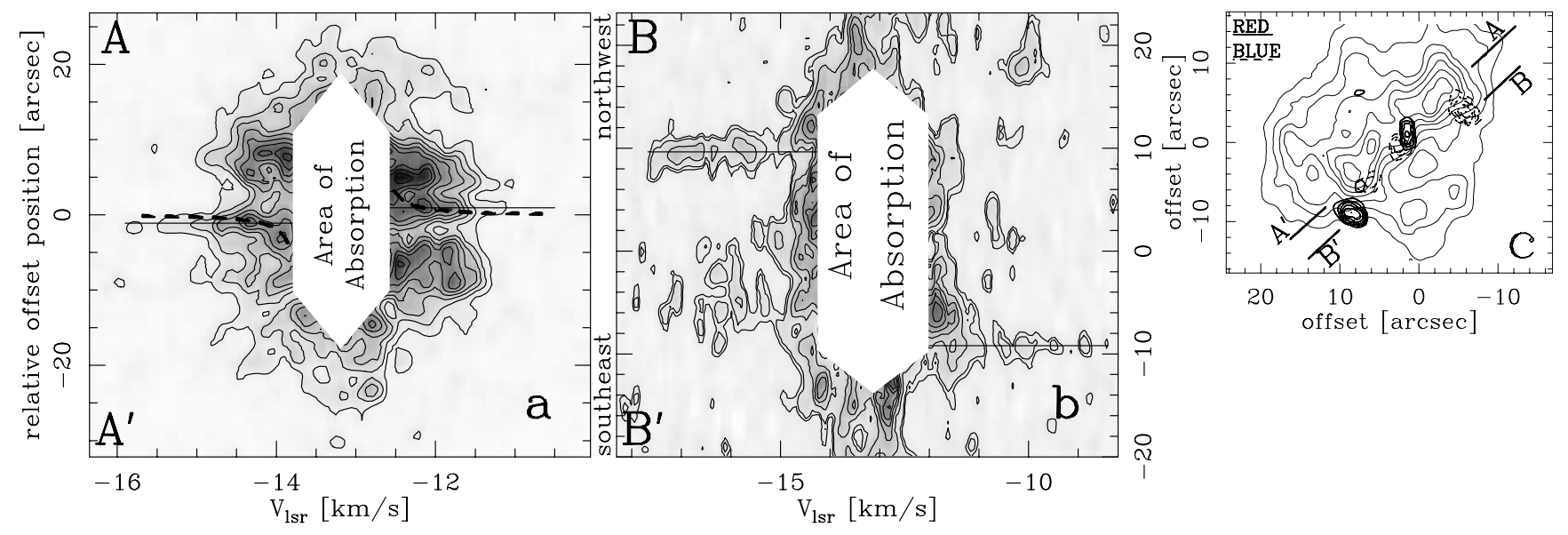

Fig. 6. a) Position-velocity diagram along the line $\mathrm{A}-\mathrm{A}^{\prime}$. The thick dashed lines indicate the curves for a simple model of Keplerian motion fitted to the outer parts of the diagram with $M_{\star}=M_{\text {disk }}=8( \pm 1) M_{\odot}$ and an inclination angle of $20^{\circ}$. For more details see Sect. 5.2. b) Position velocity map B-B' is shown. The thin straight lines in Fig. a) and b) indicate gas in a narrow sky region which show a broad velocity range. c) Same as Fig. 4b, with the cut lines of the position velocity maps indicated.

The disk mass is comparable with the mass of the star, which raises the question of dynamical stability, as measured through Toomre's $Q$ parameter (see Stone et al. 2000). Using radii between 300 and $4000 \mathrm{AU}$, temperatures of 50 to $200 \mathrm{~K}$ and the mass estimates summarized in Table $4\left(3 \ldots 18 M_{\odot}\right)$, we obtain $Q<0.5$, indicating that the disk is locally unstable. In the next section, we will demonstrate that the $3 \mathrm{~mm}$ continuum emission and the virial estimate of the deconvolved source point to a somewhat lower disk mass of 2-6 $M_{\odot}$. Considering only the innermost disk part $(r=300 \ldots .500 \mathrm{AU})$ inside a larger gas torus $(\leq 4000 \mathrm{AU})$, and using a mass of $2 M_{\odot}$ and a temperature of 100 to $200 \mathrm{~K}$, the parameter $Q$ is $\approx 0.7$, close to unity. In this case, $M_{\text {disk }} / M_{\star}=0.25$, close to the regime of $M_{\text {disk }}<0.3 M_{\star}$ where accretion disks become gravitationally stable in a global sense (Shu et al. 1990).

Hollenbach et al. (1994) propose the existence of massive disks orbiting young massive stars in the first $\approx 10^{5}$ years of their main-sequence lifetime to explain the large number of observed UCHII regions. Based on the observations of more envolved Be-stars, we know that these objectes have no disks anymore (Natta et al. 1997; Yorke et al. 1995). If we assume that these objects are $10^{5}-10^{6}$ yrs old then the disks seems to disappear after few $10^{5}$ yrs. About the destruction mechanisms, we can only speculate.

To estimate the lifetime of the disk against photoevaporation, we use the weak wind model of Hollenbach et al. (1994). For a stellar mass of $8 M_{\odot}$ and a Lyman continuum flux of $3 \times 10^{44} \mathrm{~s}^{-1}$ (Thompson 1984), the mass loss rate is $6 \times 10^{-8} M_{\odot} \mathrm{yr}^{-1}$. A $6 M_{\odot}$ disk thus takes $10^{8} \mathrm{yr}$ to destroy, making this process irrelevant.

Another mechanism to destroy the disk would be its accretion onto the central star. The accretion time scale, which is long compared with the Kelvin-Helmholtz time of the contraction (Stahler et al. 2000), can be calculated by $t_{\text {acc }} \equiv M_{\star} / \dot{M}$. Using $\dot{M}=1 \times 10^{-5} M_{\odot} \mathrm{yr}^{-1}$ (Palla \& Stahler 1992) and $M_{\star}=8 M_{\odot}$, the accretion time is $8 \times 10^{5} \mathrm{yrs}$. This time estimate is large compared with the dynamical timescale of the large-scale high-velocity outflow of $1.8 \times 10^{4}$ yrs determined by Churchwell (1999). To build up the current stellar mass of $8 M_{\odot}$, the accretion rate must have been larger in the past. Therefore, the star may still be accreting, as also suggested by the near-infrared variability of the source.

However, how long can such a massive disk exist without disrupting under its own self-gravity? Adams et al. (1989) found that disks with $M_{\star} \approx M_{\text {disk }}$ can be gravitationally unstable to eccentric matter displacements that have growth times comparable to the orbital period of the outer disk edge $\left(10^{3}-10^{4} \mathrm{yrs}\right)$. The evolution of disks having $Q_{\min } \approx 1$ was studied by Laughlin \& Bodenheimer (1994), who reported rapid fragmentation within a dynamical time scale $\left(\approx 10^{3} \mathrm{yrs}\right)$ in the inner regions of the disk. This rather short destruction time scale indicates that gravitational instabilities will be the most important destruction mechanism for more massive circumstellar disks around massive young stars. More precise disk lifetime estimates require detailed hydrodynamical simulations.

\section{3. $\mathrm{H}_{2}$ column density and mass determination}

The continuum flux and the CS line width can be used to estimate the mass of the emitting region traced by the PdB interferometer, especially the mass of the point source. Applying the formulae given by Henning et al. (2000) for the $3 \mathrm{~mm}$ continuum, the gas mass of the point source is $M_{\mathrm{gas}}^{3 \mathrm{~mm}} \approx 3 \ldots 6 M_{\odot}$. Here, a gas-to-dust mass ratio of 100 and a mean temperature of $100 \ldots 150 \mathrm{~K}$, typical for the mass-averaged temperature of a disk, is used. For the mass absorption coefficient of the dust $\kappa_{\mathrm{m}}^{\mathrm{d}}$, the value was extrapolated to $\kappa_{\mathrm{m}}^{\mathrm{d}}=0.9 \mathrm{~cm}^{2} \mathrm{~g}^{-1}$ for the wavelength of $3 \mathrm{~mm}$ (see Ossenkopf \& Henning 1994) considering densities between $10^{6}$ and $10^{8} \mathrm{~cm}^{-3}$ and a situation without icy mantles on the grains. The sourceaveraged molecular hydrogen column density for the continuum point source was determined following Henning et al. (2000) to $N\left(\mathrm{H}_{2}\right)_{(3 \mathrm{~mm})}=2.0 \ldots 3.0 \times 10^{23} \mathrm{~cm}^{-2}$, so the dust has an optical depth of $\sim 10^{-2}$ at $3 \mathrm{~mm}$ wavelength. The volume-averaged hydrogen number density $n\left(\mathrm{H}_{2}\right)$ is $2.5 \ldots 3.8 \times$ $10^{6} \mathrm{~cm}^{-3}$ for the area of the continuum emission $(>3 \sigma$; mean 
Table 4. Mass estimates from the interferometer measurements.

\begin{tabular}{lcc}
\hline \hline estimate using: & Mass $\left[M_{\odot}\right]$ & applied parameters \\
\hline \multicolumn{3}{c}{ point source } \\
\hline 3 mm continuum & $3 \ldots 6$ & $T_{\text {kin }}=150 \ldots 100 \mathrm{~K}$ \\
virial (convolved) & $13 \ldots 18$ & $\Delta v=2.5 \mathrm{~km} \mathrm{~s}^{-1}, p=1.5 \ldots 2$ \\
virial (deconvolved) & $2 \ldots 3$ & $\Delta v=2.5 \mathrm{~km} \mathrm{~s}^{-1}, p=1.5 \ldots 2$ \\
\hline \multicolumn{3}{c}{ inner envelope $\left(r \approx 25^{\prime \prime}\right)$} \\
\hline PdB CS emission & 45 & $\Delta v=1.8 \mathrm{~km} \mathrm{~s}^{-1}, T_{\text {kin }}=30$ \\
virial & 63 & $\Delta v=1.8 \mathrm{~km} \mathrm{~s}^{-1}, p=1$ \\
\hline
\end{tabular}

radius $3590 \mathrm{AU}$ ) and $0.8 \ldots 1.2 \times 10^{9} \mathrm{~cm}^{-3}$ for the deconvolved source (radius $520 \mathrm{AU}$ ), assuming that the source has the same extent along the line of sight as in the plane of the sky.

We now consider the mass of the CS emitting region. Using the PdB measurements, we can estimate the gas mass applying the formula given by Nakamura et al. (1991):

$$
\begin{aligned}
M\left(\mathrm{H}_{2}\right)\left[M_{\odot}\right]= & 4.1 \times 10^{-17}(\mathrm{D}[\mathrm{pc}])^{2} \quad S_{v} \Delta v\left[\mathrm{Jy} \mathrm{km} \mathrm{s}^{-1}\right] \\
& \times \frac{1}{X(\mathrm{CS})} \frac{\exp \left(2.4 / T_{\text {kin }}\right)}{\left(1-\exp \left(-4.7 / T_{\text {kin }}\right)\right)}
\end{aligned}
$$

Although the assumptions of LTE and an optically thin CS line emission are not met in our case, we can estimate a lower mass limit. In a sky area of 0.4 square arcmin, which is equal to a circle with a radius of $r=21.4^{\prime \prime}$, the integrated line emission $(\geq 3 \sigma)$ is $146 \mathrm{Jy} \mathrm{km} \mathrm{s}^{-1}$. Applying this formula, the mass amounts to $M_{\mathrm{gas}}^{\mathrm{H}_{2}} \geq 45 M_{\odot}$ using an abundance value of $X(\mathrm{CS})=$ $1 \times 10^{-9}$ (Sect. 7) and a mean kinetic temperature of $T_{\text {kin }}=$ $30 \mathrm{~K}$.

In addition, we can estimate the virial gas mass in the area of the continuum point source as well as in the area of the CS emission $(\geq 3 \sigma)$ applying $M_{\mathrm{vir}}=1042 \mathrm{R}[\mathrm{pc}]\left(\Delta v\left[\mathrm{~km} \mathrm{~s}^{-1}\right]\right)^{2} \alpha^{-1}$ (see Henning et al. 2000) with $\alpha=(1-p / 3) /(1-2 p / 5)$ and a density gradient of $p=1$ in the area of CS and $p=2$ in the area of the continuum source (Sect. 6.1.2).

In the area of the continuum source $(\geq 3 \sigma)$ the virial mass amounts to $M_{\text {gas }}^{\text {vir }} \approx 13 \ldots 18 M_{\odot}($ convolved size $)$ or $\approx 2 \ldots 3 M_{\odot}$ (deconvolved size) using a mean half maximum linewidth of $\Delta v_{\text {mean }}=2.5 \mathrm{~km} \mathrm{~s}^{-1}$.

For the total area of the CS emission $(\geq 3 \sigma)$, we obtain a mass of $63 M_{\odot}$ based on a $\Delta v_{\text {mean }}=1.8 \mathrm{~km} \mathrm{~s}^{-1}$. All virial masses match the mass estimates obtained from CS and from the continuum data quite well, although we have to keep in mind that virial equilibrium may not hold for the compact source.

All mass estimates are summarized in Table 4. We should note that the mass estimates for the region of the continuum source agree well with the dynamical mass of the best fits of the simple disk model in Sect. 5.4. In addition, the total gas mass traced with PdB agrees with single-dish estimates found for a similar sky region (see Sect. 4).
Table 5. Best fit results of the $\lambda 3 \mathrm{~mm}$ continuum visibilities for some selected input models.

\begin{tabular}{lrll}
\hline \hline source model & $\begin{array}{r}\text { fitted flux } \\
{[\mathrm{mJy}]}\end{array}$ & $\begin{array}{l}r_{\text {in }} \\
{\left[{ }^{\prime \prime}\right]}\end{array}$ & $\begin{array}{l}r_{\text {out }} \\
{\left[{ }^{\prime \prime}\right]}\end{array}$ \\
\hline point source + & 135.5 & & \\
Gaussian shell & 88.9 & & 0.12 \\
Gaussian shell + & 135.1 & & 0.09 \\
Gaussian shell & 73.2 & & 3.5 \\
power law $1 / r^{3}+$ & 136.4 & & 0.01 \\
Gaussian shell & 72.9 & & 3.5 \\
Gaussian shell + & 139.6 & & 0.19 \\
ring & 39.5 & 0.5 & 4.4 \\
ring + & 141.2 & 0.21 & 0.24 \\
ring & 37.9 & 0.72 & 4.6 \\
\hline
\end{tabular}

\subsection{Outflow systems and low-mass objects}

Figure 4 shows red- and blue-shifted gas blobs in the envelope region around AFGL 490 in addition to the gas associated with the dominating B-type star. Two symmetrically located gas blobs, one red-shifted and one blue-shifted, were found at a distance of about $19400 \mathrm{AU}$ from each other, centered at a position roughly $3^{\prime \prime}$ to the south of AFGL 490. The morphology of these blobs suggests that a deeply embedded young lowmass object powers a bipolar jet which enters the denser envelope material at a distance of $\sim 9700$ AU. However, all available images from the near-infrared to the radio wavelengths with the current resolution show no object at the possible position which is indicated in Fig. 5a. Cut B-B' in Fig. 6b crosses the two gas blobs of a possible jet. Both gas blobs show a velocity dispersion of at least $\Delta v=3 \mathrm{~km} \mathrm{~s}^{-1}$. Note that the "blue" gas blob coincides well with the light bow present in the $K$-band image (see Figs. 3a and 4b).

Two additional outflow systems in the surroundings of AFGL 490 were found (Fig. 4b: Ouflow systems I and II). The outflow system I seems to be driven by a low-luminosity infrared source, present in the $K$-band image of Hodapp (1994).

In general, we can assume that the complicated blue- and red-shifted gas structure found with single-dish observations (see Sect. 4) in the envelope of AFGL 490 is a superposition of different outflow systems produced by a number of lowluminosity objects. However, their influence on the general structure of the envelope around AFGL 490 seems to be small.

\section{Modelling of the envelope}

The aim of this section is to find a consistent model for the envelope, which fits both the continuum observations and the molecular line measurements (see also van der Tak et al. 2000b). Such an envelope model is needed for the understanding of the source structure, which helps in determining the evolutionary state of the object and was necessary for the interpretation of the PdB data.

Although there are a number of points which speak against a "large-scale" spherically symmetric envelope (e.g., clumpy 


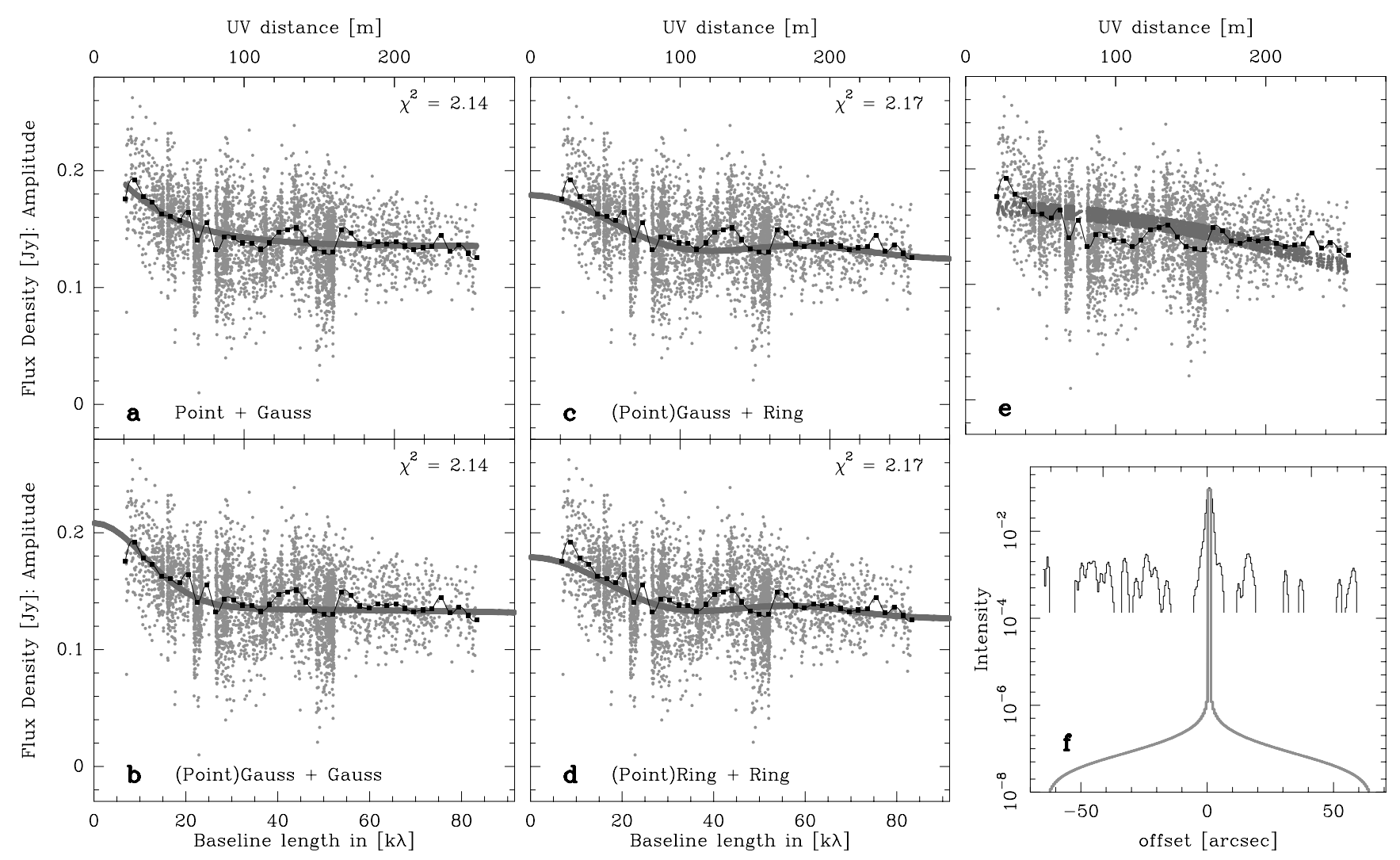

Fig. 7. a)-d) Fit results of the $\lambda 3 \mathrm{~mm}$ continuum visibilities. The grey points indicate the observations and the black squares the averages. The overplotted fit curves are presented a) for a point source and a Gaussian envelope, b) for a very narrow Gaussian source like a point source and a more extended Gaussian source, $\mathbf{c}$ ) for a very narrow Gaussian source (point-like) surrounded by a more extended ring, and d) for a very small ring source (point-like also surrounded by a more extended ring. e) PdB visiblities compared to the power law model with $\alpha=1.0$ in Sect. 6 based on the observed uv tracks. f) Cuts through the observed and the calculated maps.

structures found by Mitchell et al. 1992, 1995, broad outflow lobes, a bar-like structure in the immediate environment of AFGL 490), we will use a 1-D model for the envelope, since most of the dust continuum maps (e.g. Chini et al. 1991; van der Tak et al. 2000b) as well as maps of the total integrated line intensities imply a sufficiently spherically symmetric morphology considering beamsizes of IRAM and JCMT. The 1-D model is not meant to describe the source in detail, but is useful to measure the envelope mass at each temperature and density. The model describes the gross properties of the envelope, and is general enough that the results can be compared to those for other objects. In general, the described model will be similar to that reported by van der Tak et al. (2000b), however in this paper, more weight will be given to the modelling of the SED as well as to determine the limits of the 1-dimensional treatment of the envelope model.

\subsection{Dust continuum radiative transfer}

In order to fit the continuum measurements, we used the radiative transfer code developed by Manske et al. (1998). This is an accelerated version of the 2-D ray-tracing code developed for disk configurations by Men'shchikov \& Henning (1997). For AFGL 490, we applied this code in the 1-D version. This code is able to treat quantum heating of very small grains.
However, the lack of PAH emission in the SED, in particular in the ISO spectrum, implies that there is no need to include this heating mechanism in our case.

\subsubsection{Model parameters}

Envelope Model: based on the observations, there are some constraints for the continuum model. A main parameter of the model is the optical depth of the envelope at a standard wavelength $\left(\tau_{550} \mathrm{~nm}\right)$. From hydrogen recombination lines, Alonso-Costa \& Kwan (1989) obtained an optical extinction of $A_{\mathrm{v}}=35( \pm 5)$ mag. The optical depth at the centre of the silicate feature $\tau_{\mathrm{Si}}=2.4$ (Henning et al. 1984) and the relation $A_{\mathrm{v}} / \tau_{\mathrm{Si}}=12.7( \pm 1.6)$ mag (Rieke \& Lebofsky 1985) lead to $A_{\mathrm{v}}=$ $30( \pm 4) \mathrm{mag}$. Therefore, the $\tau_{550 \mathrm{~nm}}$ value in our models should be around this value.

To estimate the radius of the envelope, we considered different continuum and line maps for AFGL 490 published in the literature and presented in this paper as well as IRAS maps. The diameters of the molecular line maps of $80 \mathrm{arcsec}$, where the integrated surface brightness drops to $50 \%$ of the maximum, agree well with the results obtained by van der Tak et al. (2000b). Other CO observations obtained by Lada \& Harvey (1981); Snell et al. (1984) and Mitchell et al. (1995) imply that at diameters up to $\approx 100-120$ arcsec, temperature and 


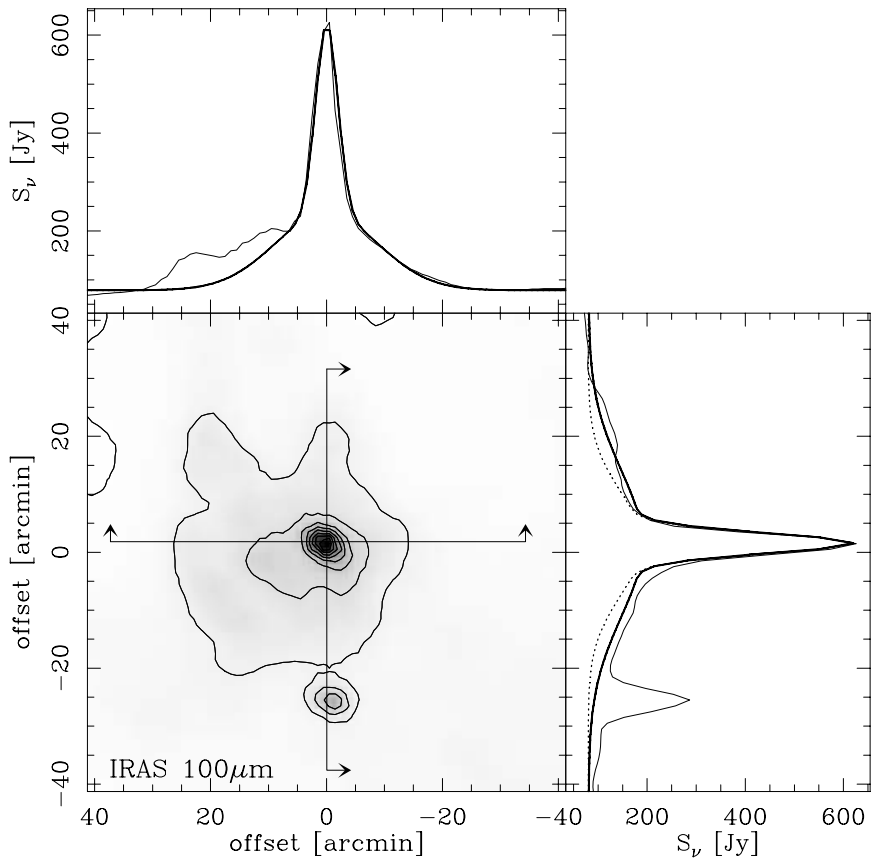

Fig. 8. Large-scale image of the IRAS $100 \mu \mathrm{m}$ map. Cuts across the maximum of the map are shown to the right and on the top. These cuts imply the presence of a compact core and a more extended envelope.

density drop below the excitation requirements for these lines. The large-scale IRAS maps reveal a dense envelope which is surrounded by rather extended emission of low intensity (Fig. 8). The map gives an averaged beam-deconvolved core diameter of $2-4$ arcmin and an envelope extension of $\approx 55$ arcmin. For the modelling, we will mainly consider the mass inside the compact IRAS source (see Fig. 8) which we will denote with "envelope of AFGL 490" hereafter. The more extended "IRAS" low-intensity emission around this envelope will play mainly a role for the interpretation of the low-excitation molecular lines, in particular, for $\mathrm{CO}$.

The value of the outer radius $r_{\text {out }}$ was constrained in our models by ISO photometry, the flux values at 870 and $1300 \mu \mathrm{m}$ and the map extensions at these wavelengths as obtained by Chini et al. (1991). In addition, we took the results of line maps, available in the literature (see Sect. 2) as well as presented in this paper, into account. Values between $70^{\prime \prime}$ and $150^{\prime \prime}$ seem to be reasonable. In all models, we used a value of $r_{\text {out }}=70^{\prime \prime}$.

The radial density structure is defined by a power law $n=$ $n_{\text {out }}\left(r / r_{\text {out }}\right)^{-\alpha}$. In a first step, we applied a constant $\alpha$ value over the envelope. In a second step, two power laws have been used with a steeper density gradient in the innermost parts and a flatter gradient in the outer regions of the envelope. This choice was motivated by the fact that the inner part of the envelope continues into the disk, whereas the outer envelope is associated with a larger-scale molecular cloud.

The inner radius $r_{\text {in }}$ of the envelope model depends on the sublimation temperatures of the dust grains which were set to be $1800 \mathrm{~K}$ for all dust components. The values of $r_{\text {in }}$ range between 7 and $15 \mathrm{AU}$. All fits were performed for a distance of $1 \mathrm{kpc}$.
Table 6. Best SED fits for the environment of AFGL 490.

\begin{tabular}{lll}
\hline \hline \multicolumn{3}{c}{ Model using a single density gradient } \\
\hline density gradient & $\alpha$ & 1.0 \\
envelope mass & $M$ & $60 M_{\odot}$ \\
inner radius & $r_{\text {in }}$ & $11 \mathrm{AU}$ \\
outer radius & $r_{\text {out }}$ & $7 \times 10^{4} \mathrm{AU}$ \\
luminosity of the star & $L_{\star}$ & $4 \times 10^{3} L_{\odot}$ \\
optical depth & $\tau_{550 \mathrm{~nm}}$ & 29.4 \\
averaged temperature at $r_{\text {out }}$ & $\bar{T}\left(r_{\text {out }}\right)$ & $15 \mathrm{~K}$ \\
density at $r_{\text {out }}$ & $n\left(r_{\text {out }}\right)$ & $3.2 \times 10^{3} \mathrm{~cm}^{-3}$ \\
\hline \multicolumn{1}{c}{ Model using two density gradients } \\
\hline inner density gradient & $\alpha(1)$ & 1.6 \\
outer density gradient & $\alpha(2)$ & 0.6 \\
envelope mass & $M$ & $100 M_{\odot}$ \\
inner radius (1) & $r_{\text {in }}(1)$ & $8 \mathrm{AU}$ \\
inner radius (2) & $r_{\text {in }}(2)$ & $700 \mathrm{AU}$ \\
outer radius & $r_{\text {out }}$ & $7 \times 10^{4} \mathrm{AU}$ \\
luminosity of the star & $L_{\star}$ & $2.2 \times 10^{3} L_{\odot}$ \\
optical depth & $\tau_{550 \mathrm{~nm}}$ & 39.5 \\
averaged temperature at $r_{\text {out }}$ & $\bar{T}\left(r_{\text {out }}\right)$ & $18 \mathrm{~K}$ \\
density at $r_{\text {out }}$ & $n\left(r_{\text {out }}\right)$ & $7.8 \times 10^{3} \mathrm{~cm}^{-3}$ \\
\hline & & \\
& &
\end{tabular}

In addition, ice absorption features of $\mathrm{H}_{2} \mathrm{O}, \mathrm{CO}$ and $\mathrm{CO}_{2}$ seen in the ISO spectra (see Sect. 7.3) indicate dust temperatures below $20 \mathrm{~K}$ at the outer radius of the envelope. This condition is met in most of our models.

The central star was assumed to be a B 3-B 2 ZAMS star. Whereas the SED fits with a constant $\alpha$ value require a somewhat higher luminosity of $L_{\star}=4000 L_{\odot}$, the models applying two density gradients are in better agreement with the observations using $L_{\star}=2200 L_{\odot}$.

We searched through the "parameter space" of $\alpha$, the mass $M$, and $r_{\text {out }}$ in order to constrain the temperature distribution which is needed for the line fits. We studied the grid $\alpha=$ $0.5,1.0,1.5,2.0, M=30,50,90,150,200,250,300,500 M_{\odot}$, and $r_{\text {out }}=40^{\prime \prime}, 70^{\prime \prime}, 100^{\prime \prime}, 180^{\prime \prime}$ for an overview of the fit results. The calculations were performed with 95-130 frequency grid points and $90-100$ radial grid points. For more than 50 radial grid points and 80 frequency grid points, the results are numerically stable.

Dust model: In several test runs, different dust populations were considered. They are all composed of an amorphous silicate and a carbon modification. For the silicate $(\mathrm{MgFeSiO}(4))$, we used the optical data published by Dorschner et al. (1995). In the case of carbon, we applied the data of four modifications: carbonaceous dust analogues produced by (a) carbonization of cellulose at $400{ }^{\circ} \mathrm{C}$, (b) carbonization of cellulose at $1000{ }^{\circ} \mathrm{C}$ (Jäger et al. 1998), (c) amorphous carbon (Preibisch et al. 1993), and (d) graphite (Draine 1985). Ice mantles were not included.

The grains are assumed to be of spherical shape with different radii $a$. The power-law index $q$ of the grain size distribution $N(a) \propto a^{-q}$ was set to $q=3.5$ (MRN: Mathis et al. 1977). The radii of grains range between $0.01 \mu \mathrm{m}$ and $2 \mu \mathrm{m}$, 


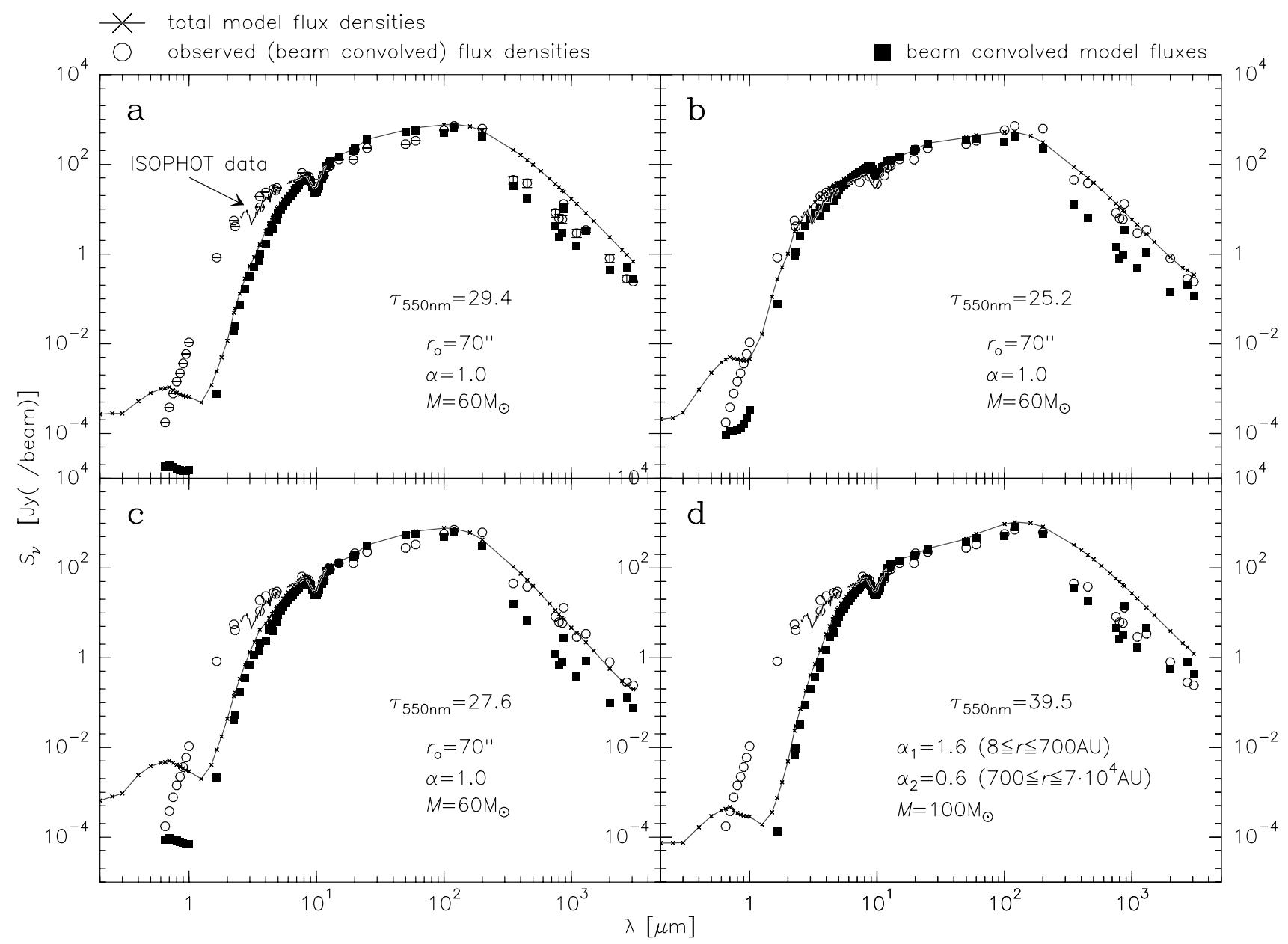

Fig. 9. a)-d) Fits of the SED. In panels a-c), the calculations were performed using the same physical structure $\left(r_{\text {out }}=70^{\prime \prime}, M=60 M_{\odot}\right.$, $\alpha=1.0$ ), but different dust compositions. The dust is composed of silicates and carbon with a mass ratio of 6:4. Optical data for silicates were taken from Dorschner et al. (1995), and different data sets were used for carbon: a) amorphous carbon from Preibisch et al. (1993), b) carbonaceous dust analogues from Jäger et al. $\left(1998,400^{\circ} \mathrm{C}\right)$, and c) the same, only produced at $1000^{\circ} \mathrm{C}$. d) Example of an SED fit using two density gradients: a steeper density gradient of $\alpha=1.6$ in the inner part ( $r \leq 700 \mathrm{AU}$ ) and a flatter gradient with $\alpha=0.6$ in the outer region (700 $\mathrm{AU} \leq r \leq 7 \times 10^{4} \mathrm{AU}$ ) of the envelope.

and the grain densities were fixed to $3.7 \mathrm{~g} \mathrm{~cm}^{-3}$ for silicates and $2.3 \mathrm{~g} \mathrm{~cm}^{-3}$ for all carbon particles. Based on an assumed total gas:dust mass ratio of 100:1, the single dust components have different mass fractions. An example is the choice of $6 \times$ $10^{-3}$ for silicates and $4 \times 10^{-3}$ for carbon. Hereafter, we abbreviate this with $\mathrm{Si}: \mathrm{C}=6: 4$. This ratio was used for most of the models, whereby the somewhat larger amount of silicate compared with carbon improved the match to the data, in particular, the fit of the $10 \mu \mathrm{m}$ absorption feature.

In the calculations, the energy balance equation is solved self-consistently and results in different temperatures for every grain component of a certain size and composition. For each material an upper and a lower temperature limit is determined.

\subsubsection{Results}

The model calculates total flux densities and apertureconvolved flux values which were compared with observations for AFGL 490 by Chini et al. (1991), Sandell (1994), Gezari et al. (1993), IRAS and ISO (Table 3, $T_{\mathrm{bb}}=400 \mathrm{~K}$ ). They are

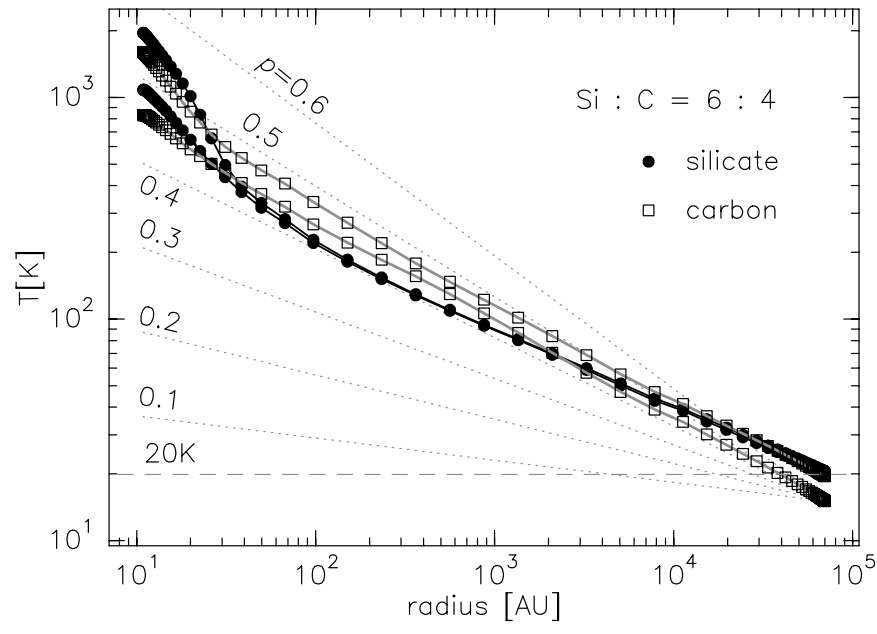

Fig. 10. The temperature distribution of the dust particles (silicates and carbon) found in most of the well fitted SEDs. Upper and lower temperature limits refer to the smallest and largest carbon and silicate dust particles, respectively. For comparison, various power-laws of $r^{-p}$ are indicated in grey for $p=0.1,0.2,0.3,0.4,0.5$, and 0.6 . 
summarized in the SEDs shown in Fig. 9. A very important result of the calculations is that the continuum models are very sensitive to the adopted dust composition. Fit results using different dust models for the same physical structure (mass, density gradient and outer radius) are shown in Figs. 9a-c. The general study of the parameter space implied that the optical data for carbonaceous grains of Preibisch et al. (1993) give mostly the best fit results. Therefore, we will constrain our further discussion on this grain model.

The consideration of different density gradients (in combination with different dust models) leads to the conclusion that the best value for the density exponent $\alpha$ is $1.0( \pm 0.2)$, using only one gradient for the whole envelope. With a shallower density gradient of $\alpha=0.5$ or a steeper gradient of $\alpha=1.5$, better fits to the observed SED simultaneously in the mid-infrared and at longer wavelengths are not possible.

The "best" model SED using a single density gradient is shown in Fig. 9a and the resulting parameters are summarized in Table 6. The fit is good in the mid- $(8-20 \mu \mathrm{m})$ and farinfrared (80-200 $\mu \mathrm{m})$ wavelength regions, and for millimeter measurements with beamsizes larger than $20^{\prime \prime}$. The fit is less good in the wavelength range $20-80 \mu \mathrm{m}$ and in the millimeter wavelength range for small beam sizes $\left(<20^{\prime \prime}\right)$, and bad at near-infrared wavelengths. In none of the models, a good fit for wavelengths $<5 \mu \mathrm{m}$ can be obtained (see also Chini et al. 1991), strongly pointing to the importance of scattered light and additional hot dust not covered in 1-D modelling. In addition, flux values at $\lambda>350 \mu \mathrm{m}$, observed with larger apertures, seem to be better fitted than the observations by Sandell (1994) using much smaller apertures. A non-spherical source structure on small scales may cause these differences.

In order to improve the fit in the wavelength range 20-100 $\mu \mathrm{m}$, we used two shells with different values of $\alpha$. One example is shown in Fig. 9d. The common property of all these models is that we need a steep density gradient in the immediate environment of AFGL 490 to produce enough near-/mid-infrared continuum emission and a shallow density gradient in the outer region of AFGL 490 to fit both the near-/ mid-infrared emission and the fluxes at longer wavelengths. In addition, a somewhat lower luminosity (between 2-3 $\times$ $10^{3} L_{\odot}$ ) gives resonable results which leads to a higher optical depth at $550 \mathrm{~nm}$, although the silicate absorption is well fitted. In general, models with two gradients fit better the wavelength range between 20 and $100 \mu \mathrm{m}$ and are, therefore, a somewhat better representation of the total SED than obtained by van der Tak et al. (2000b). The submillimeter part of the spectrum is unaffected. Note that the steeper density gradient is applied in a region with a radius of $\leq 1000 \mathrm{AU}$ where the disk is located.

One of the main reasons to fit the SED was to constrain the temperature distribution and use such a distribution as input for line calculations. Most of the models, using density gradients between $\alpha=0.5$ and 1.5 and various dust compositions, show that the calculated temperatures drop down to 15-20 K, which is consistent with the results found by the ISO-SWS measurements (see Sect. 7.3). The mean temperature distribution follows an $r^{-p}$ power law with $p=0.4( \pm 0.08)$ for radial distances of $r \geq 3-5 \times 10^{2}$ AU (Fig. 10). This is very similar to the

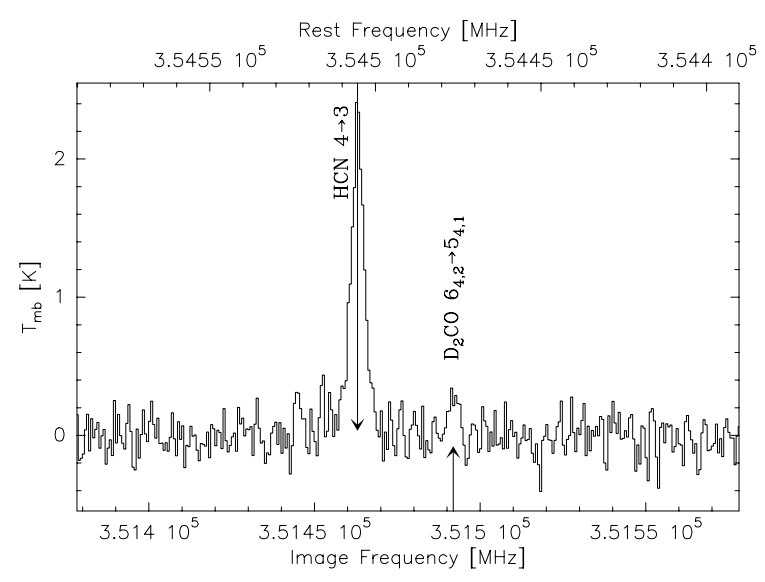

Fig. 11. Spectrum of $\mathrm{HCN} J=4 \rightarrow 3$ and a possible line of $\mathrm{D}_{2} \mathrm{CO}$.

temperature distribution used in the line transfer calculations performed by van der Tak et al. (2000b). For the smallest radii $\left(r \leq 3-5 \times 10^{2} \mathrm{AU}\right)$, a steeper gradient is obtained which agrees well with the temperature gradient of $p=0.75$, typically assumed in theoretical disk models (e.g., Hartmann 1998). Because the global temperature distribution is very similar to the line model of van der Tak et al. (2000b), this model will be used for the interpretation of the line data and the calculation of total column densities, necessary for the discussion of the chemical state of the object discussed in Sect. 7.

\subsection{Discussion of the envelope model}

Although most of the single-dish maps obtained with beamsizes $\geq 7^{\prime \prime}$ show a spherically symmetric envelope structure, there are clear signs that a 1-D model does not sufficiently fit the observational set of line and continuum data (see also van der Tak et al. 2000b). This is in agreement with the fact that we see strong evidence for a disk around AFGL 490 (see Sect. 5.2). A detailed 2D continuum modelling remains a task of the future when detailed visibility curves and higher resolution images become available at more than one wavelength.

The model by van der Tak et al. has more mass inside a given radius than our fits to the SED indicate. Inside a radius of $70^{\prime \prime}$, the model by van der Tak et al. contains $270 M_{\odot}$ vs. 60-100 $M_{\odot}$ in our model for the continuum. For this large mass, it is impossible to get the low $\tau_{550 \mathrm{~nm}}$ value of about 30 (Sect. 6.1.1), needed for the fit of the SED, especially of the silicate feature. This discrepancy may be caused by deviations from spherical symmetry. For example, the poorly collimated large-scale high-velocity outflow with a possible inclination angle between $20 \ldots 70^{\circ}$ produces two bipolar cones with large opening angles with lower density than in the surrounding envelope. If we look partly inside of such an outflow cone, the determined value $\tau_{550 \mathrm{~nm}}$ could still be around 30 even if the mass of the structure is larger than in spherically symmetric models.

Fits to the CS line emission indicate a density gradient with $\alpha=1.5$ (van der Tak et al. 2000b). The calculations in Sect. 7, which use the same model and the same radiative transfer code as described by van der Tak et al., show that lines of molecules with higher dipole moments, e.g. $\mathrm{CS}, \mathrm{H}_{2} \mathrm{CO}, \mathrm{SiO}$, and $\mathrm{HCN}$, 
require a steeper density gradient than molecules with lower values, like $\mathrm{CO}$. This can be easily understood by the different regions around AFGL 490 which are traced by the different molecules and fits well the models for the continuum.

We can summarize that all results imply a source structure where the $\mathrm{CS}$ and $\mathrm{H}_{2} \mathrm{CO}$ lines come from the innermost and densest part of the object, where the flattened bar-like structure is located. Some emission may also be contributed by the even more embedded gaseous torus/disk structure $(r<$ $4000 \mathrm{AU}=4^{\prime \prime}$ ). The $\mathrm{CO}$ and $\mathrm{CH}_{3} \mathrm{OH}$ lines as well as the continuum model support the presence of a shallower density gradient $(\alpha \approx 0.5-1.0)$ in the more extended envelope. The change of the gradient appears to be in the zone of the larger gas torus between a radius of 500 and 4000 AU. Our observations allow to conclude that the source is composed of an innermost accretion disk ( $r=50-<500 \mathrm{AU}$; see Sect. 5.2), a larger gas torus $(r<4000 \mathrm{AU})$, and a flattened dense inner envelope (the barlike structure with $r<10^{4} \mathrm{AU}$ ). Of course, these components are probably not well separated, but have smooth transitions. We should stress that the general structure of AFGL 490 is very similar to G 192.16-3.82 (Shepherd et al. 2001, see also Sect. 8).

\section{Chemistry of the envelope}

\subsection{Results from single-dish spectra}

Table 2 summarizes the line data for all species measured at the [0, 0] map position. A $2 \sigma$ upper limit is given for important diagnostic lines which were not detected. Most of the detections were fitted by a single Gaussian profile in order to estimate the values $T_{\mathrm{mb}}, \Delta v$, and $\int T_{\mathrm{mb}} \mathrm{d} v$. Clear exceptions to such pure Gaussian profiles are the ${ }^{12} \mathrm{CO}$ and ${ }^{13} \mathrm{CO}$ lines, the lowerexcitation lines of CS, and the SO lines which partly possess strong line wings.

Comparison of our much longer integrated ${ }^{13} \mathrm{CO} J=3 \rightarrow 2$ spectrum with the measurements by Mitchell et al. (1992, same beamsize) shows that the main features are similar. However, the shape of our spectrum is more comparable with that of the ${ }^{12} \mathrm{CO}$ lines. Only the line intensity ratio between the central narrow component and the broader (inner wing) component differs compared with the main isotope. The ${ }^{13} \mathrm{CO} J=3 \rightarrow 2$ observations show strong self-absorption features in a similar velocity range as ${ }^{12} \mathrm{CO}$. The ${ }^{13} \mathrm{CO}$ high-velocity outflow emission covers the velocity range $v_{\mathrm{lsr}}=41( \pm 5) \mathrm{km} \mathrm{s}^{-1}$ for $T_{\mathrm{mb}} \geq$ $2 \sigma$.

In addition, the results of our $\mathrm{H}^{13} \mathrm{CO}^{+} J=3 \rightarrow 2$ measurements agree with those by Hasegawa \& Mitchell (1995) within the calibration uncertainty of $15 \%$.

The increase of the $\mathrm{SiO}$ line intensities with higher excitation temperatures comes possibly from the fact that the JCMT beam sizes at 347 and $217 \mathrm{GHz}$ are different and the $\mathrm{SiO}$ source filling factor is (much) smaller than 1. A similar effect appears to be present in the SO lines.

Using the interstellar isotopic abundance ratios (see e.g. Wilson \& Rood 1994), a first estimate of the optical depths at the line centers is possible. Using ${ }^{12} \mathrm{C} /{ }^{13} \mathrm{C}=60 \ldots 65,{ }^{16} \mathrm{O} /{ }^{18} \mathrm{O}=$ 500 , and ${ }^{16} \mathrm{O} /{ }^{17} \mathrm{O}=2500$, our measured ${ }^{12} \mathrm{CO} J=3 \rightarrow 2,4 \rightarrow 3$,

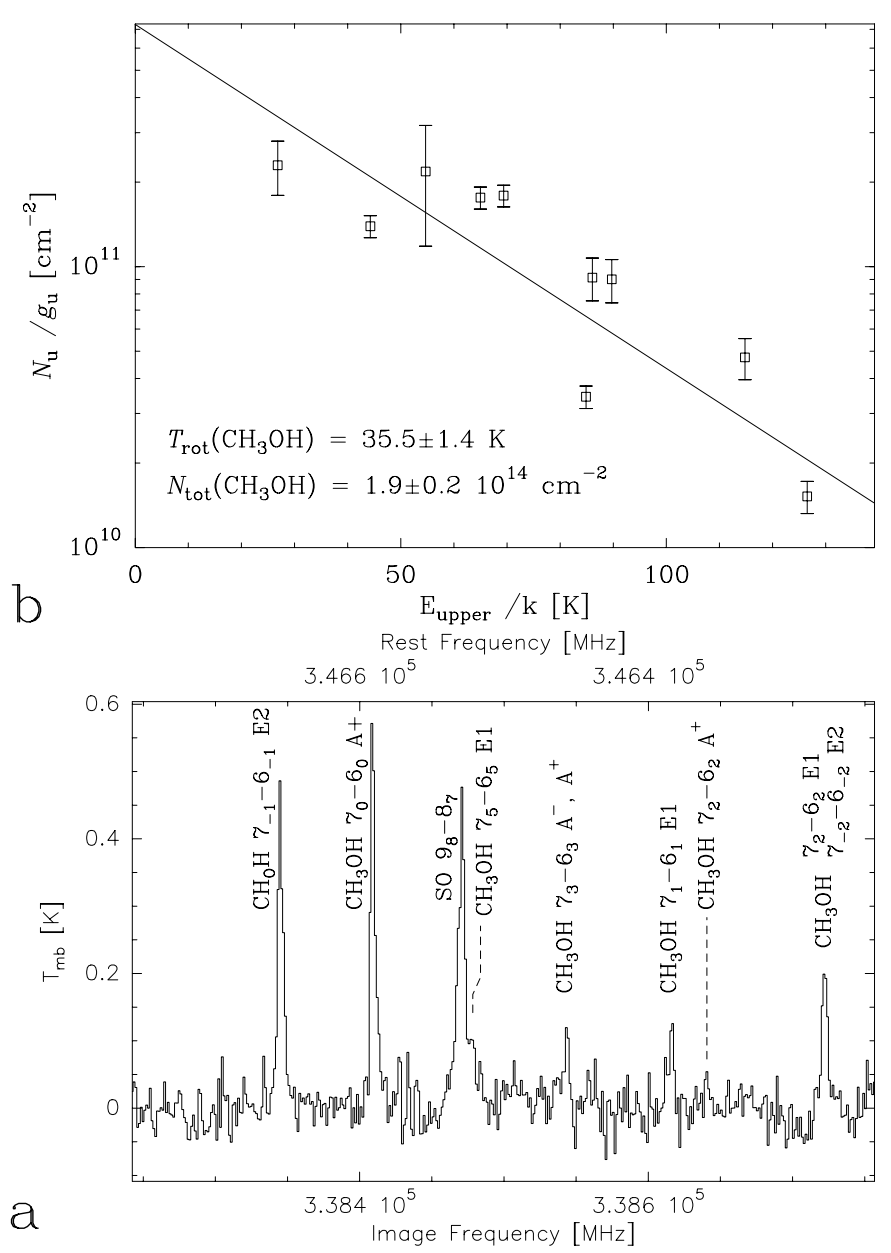

Fig. 12. a) Spectrum of the $\mathrm{CH}_{3} \mathrm{OH} J=7 \rightarrow 6$ transition. b) Rotation diagram for $\mathrm{CH}_{3} \mathrm{OH}$ toward AFGL 490.

and ${ }^{13} \mathrm{CO} J=3 \rightarrow 2$ lines (narrow and "inner wing" components) are optically thick. The line intensity ratio of $\mathrm{C}^{18} \mathrm{O} J=$ $2 \rightarrow 1$ to $\mathrm{C}^{17} \mathrm{O} J=3 \rightarrow 2$ of $\approx 3 \ldots 5$ implies that the $\mathrm{C}^{18} \mathrm{O} J=$ $2 \rightarrow 1$ transition is slightly optically thick. Considering the $\mathrm{CS}$ isotopes with a ${ }^{32} \mathrm{~S} /{ }^{34} \mathrm{~S}$ ratio of 22 , the measured ratios of 15.7 for $J=7 \rightarrow 6$ and 18.4 for $J=5 \rightarrow 4$ suggest that the CS lines are close to optically thin. An estimate of the optical depths of the $\mathrm{H}_{2} \mathrm{CO} J=3 \rightarrow 2$ transitions from the $\mathrm{H}_{2}^{13} \mathrm{CO} J=$ $3_{1,2} \rightarrow 2_{1,1}$ measurement implies that these lines as well as the higher-excitation transitions of $\mathrm{H}_{2} \mathrm{CO}$ are also optically thin. The possible detection of $\mathrm{D}_{2} \mathrm{CO}$ (Fig. 11) is of interest because so far, this molecule has only been found in Orion (Turner 1990) and IRAS 16293-2422 (Ceccarelli et al. 1998). The possible presence of this molecule has to confirmed by the detection of other $\mathrm{D}_{2} \mathrm{CO}$ lines.

The observed transitions of $\mathrm{CH}_{3} \mathrm{OH}$ are useful as a first indicator for the overall temperature of the envelope through the rotation diagram (Blake et al. 1987; van der Tak et al. 2000a). Assuming optically thin emission and thermalized excitation, we obtain $T_{\text {rot }}\left(\mathrm{CH}_{3} \mathrm{OH}\right) \approx 35( \pm 1.4) \mathrm{K}$ and $N\left(\mathrm{CH}_{3} \mathrm{OH}\right) \approx$ $1.9( \pm 0.2) \times 10^{14} \mathrm{~cm}^{-2}$ (Fig. 12), similar (within the calibration uncertainties) to the earlier estimate by van der Tak et al. (2000a). In the likely case that $\mathrm{CH}_{3} \mathrm{OH}$ is subthermally excited, the calculated $T_{\text {rot }}$ value is an overestimation of the mean 

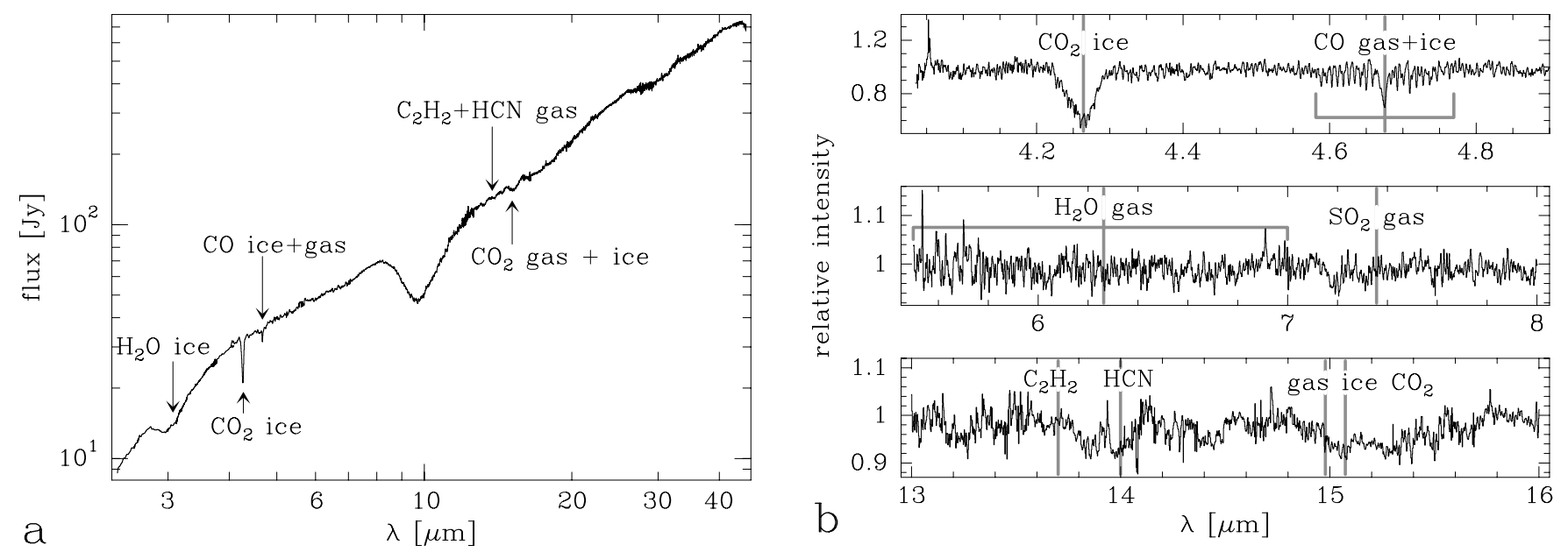

Fig. 13. a) ISO-SWS 01 spectrum toward AFGL 490 between 2.5 and $20 \mu \mathrm{m}$. The observed features of solid $\mathrm{CO}, \mathrm{CO}_{2}$ and $\mathrm{H}_{2} \mathrm{O}$ as well as gas-phase CO are indicated. b) Blow-up ISO-SWS 06 spectrum parts of the three wavelength ranges. The vertical bars indicate the positions of the strongest features.

kinetic temperature in the envelope. Thus, these data also indicate that the envelope is cold. There is no evidence for variations in the methanol abundance with radius.

\subsection{Molecular abundances}

For a detailed picture of the chemical state of the object, we applied the envelope model from van der Tak et al. (2000b) and the line radiative transfer code described in Hogerheijde \& van der Tak (2000). The model has the following parameters: an exponent of the density gradient $\alpha=-1.5$, an outer radius $r_{\text {out }}=70^{\prime \prime}$, an inner radius $r_{\text {in }}=70 \mathrm{AU}$, and 30 spherical shells spaced logarithmically within the range $r_{\text {in }}$ to $r_{\text {out }}$. A turbulent linewidth of $b_{\mathrm{D}}=1.05 \mathrm{~km} \mathrm{~s}^{-1}$ was used. Radiative pumping by dust was not included.

The data listed in Table 2 were fitted by synthetic line profiles, using molecular data from Schinke et al. (1985) for CO, Green \& Chapman (1978) for CS and $\mathrm{HC}_{3} \mathrm{~N}$, Green (1991) for $\mathrm{H}_{2} \mathrm{CO}$, Bieniek \& Green $(1980,1983)$ for SiO, Green \& Thaddeus (1974) for HCN, DCN, and CCH, Green (1989) for HDO, Green (1975) for $\mathrm{N}_{2} \mathrm{H}^{+}$, Schröder et al. (1991) for C, Monteiro (1985) and Green (1975) for $\mathrm{HCO}^{+}$, and Green (1994) for SO. For NO and NS, no collisional rate coefficients are available, while for $\mathrm{SO}_{2}$, the available numbers (Green 1995) do not cover the states observed here.

For most molecules, the observed line profiles are well fitted using a constant abundance throughout the envelope. Only for the main isotopes of $\mathrm{CS}$ and $\mathrm{HCN}$, the synthetic profiles are self-absorbed, and the abundance is mainly constrained by the isotopic lines. Similar differences between the shapes of observed and modeled optically thick lines were found toward AFGL 2591 (van der Tak et al. 1999) and likely indicate deviations from the assumed spherical geometry. Such deviations are also suggested by our failure to fit the continuum spectrum at $\lambda \leq 10 \mu \mathrm{m}$.

Table 7 lists the determined abundances. It is seen that for most species, values of $\sim 10^{-10}$ are found, except for the dominant carriers of carbon, $\mathrm{CO}$ and $\mathrm{C}$, which have much higher abundances, and for $\mathrm{SiO}$ and $\mathrm{N}_{2} \mathrm{H}^{+}$, which are less abundant. The derived abundances for the same mass of $270 M_{\odot}$ inside a radius of $r_{\text {out }}=70^{\prime \prime}$ depend somewhat on the adopted density structure: if $\alpha=1.0$ is used instead of $\alpha=1.5$, the best-fit abundances increase by factors of 5-10.

The table also compares the derived abundances to the values found in two other embedded protostellar objects. AFGL 2591 has an envelope mass similar to that of AFGL 490, but a higher luminosity; W3 IRS5 has both a higher mass and a higher luminosity. While the chemical composition of AFGL 2591 has been derived using the same method as in this section, single values for temperature and density were used for W3 IRS5. Values derived for W3 IRS5 using a model with temperature and density gradients are similar to those quoted in Table 7 for $\mathrm{CO}$ and $\mathrm{CH}_{3} \mathrm{OH}$, but an order of magnitude larger for CS and $\mathrm{H}_{2} \mathrm{CO}$ (van der Tak et al. 2000a,b). Therefore, values for other species may also need to be revised upwards. Nevertheless, the table shows that the abundances in AFGL 490 are lower than those in AFGL 2591 by factors of 10-100, but that they are within factors of a few of those in W3 IRS5.

The low molecular abundances likely reflect the cold nature of the envelope, causing an overall depletion, possibly by freeze-out onto dust grains. For the ions $\mathrm{HCO}^{+}$and $\mathrm{N}_{2} \mathrm{H}^{+}$, the low value of the cosmic-ray ionization rate in this source (van der Tak \& van Dishoeck 2000) may also play a role. The data do not show any further chemical differentiation, except that sulphur-bearing species may be underabundant. The overall molecular depletion suggests an early evolutionary stage.

\subsection{Results from ISO-SWS}

The ISO-SWS spectrum between $2.5-45 \mu \mathrm{m}$ (Fig. 13) shows almost no lines of gas-phase molecules in contrast to the spectra toward the intermediate-mass protostar $\mathrm{LkH} \alpha 225$ (van den Ancker 2000) and massive protostars (Lahuis \& van Dishoeck 2000; Boonman et al. 2000). For example, the $v_{2}$ ro-vibrational band of gas-phase $\mathrm{H}_{2} \mathrm{O}$ at $6 \mu \mathrm{m}$, which is strong toward most of the massive protostars (Boonman et al. 2000) 
Table 7. Molecular abundances relative to $\mathrm{H}_{2}$.

\begin{tabular}{|c|c|c|c|c|}
\hline \multirow[b]{2}{*}{ molecule } & \multicolumn{2}{|c|}{ AFGL $490^{a}$} & \multirow[t]{2}{*}{ AFGL $2591^{b}$} & \multirow[t]{2}{*}{ W3 IRS5 } \\
\hline & $\alpha=1.5$ & $\alpha=1.0$ & & \\
\hline $\mathrm{CO}$ & $3 \times 10^{-5}$ & $6-7 \times 10^{-5}$ & $1.5 \times 10^{-4}$ & $1 \times 10^{-4}$ \\
\hline $\mathrm{CS}$ & $7 \times 10^{-10}$ & $2-3 \times 10^{-9}$ & $1 \times 10^{-8}$ & $8 \times 10^{-10}$ \\
\hline $\mathrm{H}_{2} \mathrm{CO}$ & $2.5 \times 10^{-10}$ & $1.5 \times 10^{-9}$ & $4 \times 10^{-9}$ & $3 \times 10^{-10}$ \\
\hline SO & $3-4 \times 10^{-10}$ & $2 \times 10^{-9}$ & $2 \times 10^{-8}$ & $2 \times 10^{-8}$ \\
\hline $\mathrm{SiO}$ & $1.5 \times 10^{-11}$ & $1 \times 10^{-10}$ & $1 \times 10^{-10}$ & $2 \times 10^{-11}$ \\
\hline $\mathrm{HCN}$ & $5-7 \times 10^{-10}$ & $2-4 \times 10^{-9}$ & $2 \times 10^{-8}$ & $2 \times 10^{-9}$ \\
\hline $\mathrm{HDO}$ & $\leq 1 \times 10^{-11}$ & $\leq 8 \times 10^{-11}$ & $\ldots$ & \\
\hline $\mathrm{HC}_{3} \mathrm{~N}$ & $0.8-1 \times 10^{-10}$ & $2 \times 10^{-9}$ & $2 \times 10^{-8}$ & $\leq 4 \times 10^{-11}$ \\
\hline $\mathrm{C}_{2} \mathrm{H}$ & $8 \times 10^{-10}$ & $2 \times 10^{-9}$ & $2 \times 10^{-9}$ & $5 \times 10^{-10}$ \\
\hline $\mathrm{C}$ & $3 \times 10^{-6}$ & $7 \times 10^{-6}$ & $3 \times 10^{-6}$ & $2 \times 10^{-5}$ \\
\hline $\mathrm{HCO}^{+}$ & $8 \times 10^{-10}$ & $5 \times 10^{-9}$ & $1 \times 10^{-8}$ & $7 \times 10^{-10}$ \\
\hline $\mathrm{N}_{2} \mathrm{H}^{+}$ & $7-8 \times 10^{-12}$ & $9 \times 10^{-11}$ & $5 \times 10^{-10}$ & $\leq 6 \times 10^{-12}$ \\
\hline $\mathrm{CH}_{3} \mathrm{OH}$ & $1 \times 10^{-9}$ & $\ldots$ & $2.6 \times 10^{-9}$ & $6 \times 10^{-10}$ \\
\hline
\end{tabular}

${ }^{a}$ Values given for two values of the density law exponent for $270 M_{\odot}$ inside a radius of $r_{\text {out }}=70^{\prime \prime}$. Value for $\mathrm{CH}_{3} \mathrm{OH}$ from van der Tak et al. (2000a).

${ }^{b}$ From van der Tak et al. (2000b) (CO, CS, $\left.\mathrm{H}_{2} \mathrm{CO}\right)$; van der Tak et al. (2000a) $\left(\mathrm{CH}_{3} \mathrm{OH}\right)$; Boonman et al. (2001) (HCN); Choi et al. (1994) (C); van der Tak et al. (1999) $\left(\mathrm{HCO}^{+}\right)$; van der Tak et al. (in prep.) ( $\mathrm{SO}, \mathrm{SiO}, \mathrm{HC}_{3} \mathrm{~N}, \mathrm{C}_{2} \mathrm{H}, \mathrm{N}_{2} \mathrm{H}^{+}$). When the abundance was found not to be constant, the value in the cold outer part of the envelope is given. ${ }^{c}$ From de Boisanger et al. (1996) $\left(\mathrm{N}_{2} \mathrm{H}^{+}, \mathrm{C}\right)$; Helmich et al. (1996) (HDO); Helmich \& van Dishoeck (1997) (other molecules).

has not been detected toward AFGL 490, placing an upper limit on the total amount of gas-phase $\mathrm{H}_{2} \mathrm{O}$ present (Table 8). Since gas-phase $\mathrm{H}_{2} \mathrm{O}$ abundances are predicted to be enhanced in warm regions and shocks, this indicates that AFGL 490 is not a "hot" source throughout the envelope. This picture is confirmed by the absence of the gas-phase molecules HCN at $14.0 \mu \mathrm{m}$ and $\mathrm{C}_{2} \mathrm{H}_{2}$ at $13.7 \mu \mathrm{m}$ which trace hot dense gas (Lahuis \& van Dishoeck 2000).

The presence of the $4.7 \mu \mathrm{m}$ band of $\mathrm{CO}$ ice, which evaporates at temperatures $>20 \mathrm{~K}$, is in agreement with the cold nature of the source. This ice feature is also seen toward NGC 2264 IRS1, but is weak or almost completely absent in the hot massive protostars, such as AFGL 2591 (Tielens et al. 1991) and in $\mathrm{LkH} \alpha 225$. The bending mode of gas-phase $\mathrm{CO}_{2}$ at $15.0 \mu \mathrm{m}$ is not detected toward AFGL 490. If evaporation from ices is the dominant production mechanism for gas-phase $\mathrm{CO}_{2}$, this suggests that the temperature is below $\sim 100 \mathrm{~K}$. This is confirmed by the solid $\mathrm{CO}_{2}$ bands at 4.3 and $15.2 \mu \mathrm{m}$, which show little evidence for heating of the $\mathrm{CO}_{2}$ ice (Gerakines et al. 1999). The absence of the ro-vibrational band of gas-phase $\mathrm{SO}_{2}$ around $7.3 \mu \mathrm{m}$ and the presence of very few pure rotational $\mathrm{SO}_{2}$ lines in the submillimeter (see Table 2) do fit in this picture.

The ro-vibrational band of gas-phase $\mathrm{CO}$ at $4.6 \mu \mathrm{m}$ is clearly detected in the SWS spectrum. However these lines are saturated. Mitchell et al. (1995) have analyzed the rovibrational ${ }^{13} \mathrm{CO}$ lines in the same wavelength region at higher spectral resolution, resulting in two temperature components for the gas, one at $24 \mathrm{~K}$ and one at $107 \mathrm{~K}$, consistent with the envelope structure. The latter value has been adopted to place upper limits on the column densities of other gas-phase species, using synthetic model spectra (Helmich 1996) with a Doppler parameter $b=3-5 \mathrm{~km} \mathrm{~s}^{-1}$, which agrees well with the value of $b=3.5 \mathrm{~km} \mathrm{~s}^{-1}$ found by Mitchell et al. (1995). The resulting column densities and abundances are summarized in Table 8. In summary, the results from the ISO-SWS spectra show that most of the envelope gas and the ice surrounding AFGL 490 have temperatures below $100 \mathrm{~K}$, indicating that AFGL 490 is a source with a cold envelope. These results match very well all the other temperature estimates discussed throughout the paper.

\section{Comparison with other embedded intermediate-mass objects}

Taking all results together, we can conclude that the object AFGL 490 seems to be in a similar evolutionary state as the objects G 192.16-3.82 (Shepherd et al. 2001), IRAS 20126+4104 (Cesaroni et al. 1999; Zhang et al. 1998), Cep A HW2 (Narayanan \& Walker 1996; Gómez et al. 1999), AFGL 5142 (Hunter et al. 1999; Minier et al. 2000), and the object IRc2(I) in the Orion BN-KL region for which Gezari et al. (1998) recalculated a luminosity of only $10^{3} L_{\odot}$.

All these objects are very young deeply embedded stars (age about $10^{4}-10^{5}$ years) with masses of 8-20 $M_{\odot}$ (zero-age main sequence spectral type B0-B3) (or even larger). They show poorly collimated high-velocity bipolar outflows with no strong evidence for highly-collimated jets. All these objects show flattened rotating disk-like gas structures with diameters up to $5000 \mathrm{AU}$, which is larger than the expected size of an accretion disk, but could be the extension of an inner (accretion) disk (see also Minier et al. 2000). However, these larger gas tori can hardly be the location at which the outflow is launched (see Shepherd et al. 2001 and references therein). The origin of these outflows cannot be traced with presentday interferometers. In the innermost part of AFGL 490, we found strong evidence for a disk with dimensions similar to that of G 192.16-3.82.

The young stars are also embedded in more extended molecular envelopes. All observations of AFGL 490 point to the fact that this envelope is cold. In the envelope, several lowmass companions close to the central star are located. They seem to have a similar age as the most massive object, showing bipolar jets, highly collimated outflows as well as circumstellar disks.

Objects like NGC 2264 IRS1 appear to be in a later phase than the previously discussed sources. Here the disk/torus system has disappeared (see Schreyer et al. 2002) and the B-type star has created a hole in the centre of its envelope. An even more evolved stage could be the Herbig Be stars, where no evidence for disks exists for spectral types earlier than B7-B8 (Natta et al. 2000; Pantin et al. 2000; Grady et al. 2001).

Our chemical analysis supports these conclusions. Molecular abundances in the envelope of AFGL 490 are comparable to those in the cold envelope W3 IRS5 (Helmich \& van Dishoeck 1997), and an order of magnitude below the values in AFGL 2591 (van der Tak 1999, 2000a,b) and NGC 2264 
Table 8. ISO-SWS results.

\begin{tabular}{lccccccc}
\hline \hline Species & $\begin{array}{c}T_{\text {ex,gas }}^{a} \\
{[\mathrm{~K}]}\end{array}$ & $\begin{array}{c}N_{\text {gas }} \\
{\left[\mathrm{cm}^{-2}\right]}\end{array}$ & $\begin{array}{c}T_{\text {solid }} \\
{[\mathrm{K}]}\end{array}$ & $\begin{array}{c}N_{\text {solid }} \\
{\left[\mathrm{cm}^{-2}\right]}\end{array}$ & $N_{\text {gas }} / N\left(\mathrm{H}_{2}\right)^{b}$ & $N_{\text {solid }} / N\left(\mathrm{H}_{2}\right)^{b}$ & $N_{\text {gas }} / N_{\text {solid }}$ \\
\hline $\mathrm{H}_{2} \mathrm{O}$ & 107 & $<3 \times 10^{17}$ & - & $7.3 \times 10^{17 c}$ & $<8.0 \times 10^{-6}$ & $1.9 \times 10^{-5}$ & $<0.41$ \\
$\mathrm{CO}_{2}$ & 107 & $<1 \times 10^{16}$ & $10^{d}$ & $1.7 \times 10^{17 d}$ & $<2.7 \times 10^{-7}$ & $4.5 \times 10^{-6 d}$ & $<0.06$ \\
$\mathrm{HCN}^{16}$ & 107 & $<3 \times 10^{16}$ & - & - & $<8.0 \times 10^{-7}$ & - & - \\
$\mathrm{C}_{2} \mathrm{H}_{2}$ & 107 & $<5 \times 10^{15}$ & - & - & $<1.3 \times 10^{-7}$ & - & - \\
$\mathrm{SO}_{2}$ & 107 & $<4 \times 10^{16}$ & - & - & $<1.1 \times 10^{-6}$ & - & - \\
\hline
\end{tabular}

${ }^{a}{ }^{13} \mathrm{CO}$ temperature from Mitchell et al. (1995).

${ }^{b}$ Using the total gas-phase $\mathrm{H}_{2}$ column density $N\left(\mathrm{H}_{2}\right)=3.75 \times 10^{22} \mathrm{~cm}^{-2}$ from Mitchell et al. $(1995)$ assuming $N(\mathrm{CO}) / N\left(\mathrm{H}_{2}\right)=2 \times 10^{-4}$ and $\mathrm{CO} /{ }^{13} \mathrm{CO}=60$.

${ }^{c}$ From Willner et al. (1982).

${ }^{d}$ From Gerakines et al. (1999).

IRS1 (Schreyer et al. 1997). The low abundances of AFGL 490 and W3 IRS5 likely result from depletion onto dust grains during the cold pre-stellar stages. In the other two sources, the central star has heated the envelope to higher temperatures, causing molecules to return to the gas phase.

\section{Conclusions}

We presented a detailed investigation of the region around the luminous infrared source AFGL 490. A comprehensive set of single-dish maps in different line transitions, interferometer maps in CS $J=2 \rightarrow 1$ and the corresponding continuum at 97.98 GHz, and ISO-SWS and ISOPHOT data, allowed the development of a geometrical and evolutionary model for this source:

(1) The data strongly indicate that the central star of AFGL 490 of spectral type B2-B3 is located in a small ionized region with an accelerating stellar wind (Bunn et al. 1994) surrounded by an accretion disk with an outer radius smaller than $500 \mathrm{AU}$. The outer regions of this disk merge with a larger gas torus with $r<4000 \mathrm{AU}$. This region is surrounded by the remnant of a flattened cloud core $\left(r<10^{4} \mathrm{AU}\right)$. All these structures together are embedded in a more extended envelope. Therefore, we could call AFGL 490 a "matroschka" or the perfect example of a Russian doll. All known observational data with the present resolution do not suggest that the massive gas disk is a merger of multiple disks around multiple stars.

The gas mass inside a radius of $4000 \mathrm{AU}$ is about $8 M_{\odot}$ and similar to the mass of the central star. The mass estimates obtained for the continuum point source are somewhat lower with values between 3 and $6 M_{\odot}$ within a radius of $\leq 500$ AU. AFGL 490 resembles in many aspects the source G 192.16-3.82, where Shepherd et al. (2001) found a disk. However, AFGL 490 has the advantage that it is located at half of the distance of this object and perfectly suited for further interferometry and adaptive optics measurements at shorter wavelengths. In both sources, the disk mass is much larger than found around Herbig Ae stars by Mannings \& Sargent (1997).

(2) Radiative transfer models to fit the spectral energy distribution and the analysis of line data point to steeper density gradients $(\alpha \approx 1.5)$ in the inner disk-like dominated region ( $r$ smaller than $4000 \mathrm{AU}$ ) and a shallower gradient $(\alpha \approx 0.5-1.0)$ in the more extended envelope. In addition, the ISO-SWS data and additional molecular line data such as $\mathrm{CH}_{3} \mathrm{OH}$ measurements clearly indicate that the temperature in the outer envelope is below $100 \mathrm{~K}$. Therefore, we can conclude that we see one of the rare cases of early B stars with a disk system which is still deeply embedded in a cold molecular core.

(3) The combination of infrared images with the PdB interferometer data show that a number of other low-mass young stellar objects with outflows are embedded in the envelope of AFGL 490. The data clearly rule out that these outflows have a dramatic effect on the physical and chemical structure of the envelope.

(4) Using the results of the modelling for the temperature and density distribution, we estimate the abundances of 13 molecules towards the position of AFGL 490. The chemistry is characterized by an overall depletion of molecules by a factor of $\sim 10$, perhaps due to freezing out onto dust grains. Along with the results from our ISO-SWS data and the derived excitation temperature of $\mathrm{CH}_{3} \mathrm{OH}$, the chemical state reflects the cold conditions in the envelope of AFGL 490.

Acknowledgements. The authors are grateful to Remo Tilanus and Fred Baas for their support during the JCMT observing runs. Additionally, we thank Melvin Hoare for the $H$-band fits image and Ilaria Pascucci for support of the fit of the visibilities. This work was supported by a SPINOZA grant from The Netherlands Organization of Scientific Research (NWO). The research of the Jena group was supported by DFG grant He 1935/14-1.

\section{References}

Adams, F. C., Ruden, S. P., \& Shu, F. H. 1989, ApJ, 347, 959

Alonso-Costa, J. L., \& Kwan, J. 1989, ApJ, 338, 403

Bieniek, R. J., \& Green, S. 1980, J. Chem. Phys., 73, 851

Bieniek, R. J., \& Green, S. 1983, ApJ, 265, L29

Blake, G. A., Sutton, E. C., Masson, C. R., \& Phillips, T. G. 1987, ApJ, 315, 621

Boonman, A. M. S., van Dishoeck, E. F., Lahuis, F., Wright, C. M., \& Doty, S. D. 2000, Gas-phase $\mathrm{H} 2 \mathrm{O}$ and $\mathrm{CO} 2$ towards massive protostars, in ISO beyond the Peaks: The 2nd ISO workshop on analytical spectroscopy, ed. A. Salama, M. F. Kessler, K. Leech, \& B. Schulz, ESA-SP 456, 67 
Boonman, A. M. S., Stark, R., van der Tak, F. F. S., et al. 2001, ApJ, 553, L63

Bunn, J. C., Hoare, M. G., \& Drew, J. E., 1995, MNRAS, 272, 346

Brand, J. 1986, Ph.D. Thesis, University of Leiden

Brand, J., \& Blitz, L. 1993, A\&A, 275, 67

Campbell, B., Persson, S. E., \& McGregor, P. J. 1986, ApJ, 305, 336

Campbell, B., Persson, S. E., \& Matthews, K. 1989, AJ, 98, 643

Cesaroni, R., Felli, M., Jenness, T., et al. 1999, A\&A, 345, 949

Ceccarelli, C., Castets, A., Loinard, L., Caux, E., \& Tielens, A. G. G. M. 1998, A\&A, 338, L43

Chini, R., Henning, Th., \& Pfau, W. 1991, A\&A, 247, 157

Churchwell, E. 1999, Massive Star Formation, in The Origin of Stars and Planetary Systems, ed. C. J. Lada, \& N. D. Kylafis (Kluwer Academic Publishers), 515

Choi, M., Evans II, N. J., Jaffe, D. T., \& Walker, C. K. 1994, ApJ, 435, 734

de Boisanger, C., Helmich, F. P., \& van Dishoeck, E. F. 1996, A\&A, 310,315

Di Francesco, J., Evans II, N. J., Harvey, P. M., et al. 1997, ApJ, 482, 433

Dorschner, J., Begemann, B., Henning, Th., Jäger, C., \& Mutschke, H. 1995, A\&A, 300, 503

Draine, B. T. 1995, Properties of Dust in Diffuse Clouds, in The Physics of the Interstellar Medium and Intergalactic Medium, ASP Conf. Ser., 80, ed. A. Ferrara, C. F. McKee, C. Heiles, \& P. R. Shapiro (Astronomical Society of the Pacific, San Francisco, California), 133

Dutrey, A., Guilloteau, S., Duvert, G., et al. 1996, A\&A, 309, 493

Evans II, N. J. 1999, Star Formation at High Resolution, in Science with the Atacama Large Millimeter Array (ALMA), Associated Universities, Carnegie Institution of Washington, 17

Frerking, M. A., Langer, W. D., \& Wilson, R. W. 1982, ApJ, 262, 590

Gear, W. K., Gee, G., Robson, E. I., Ade, P. A. R., \& Duncan, W. D. 1986, MNRAS, 219, 835

Gerakines, P. A., Whittet, D. C. B., Ehrenfreund, P., et al. 1999, ApJ, 522,357

Gezari, D. Y., Schmitz, M., Pitts, P. S., \& Mead, J. M. 1993, Catalog of Infrared Observations, 3rd ed., NASA Ref. Publication 1294

Gezari, D. Y., Backman, D. E., \& Werner, M. W. 1998, ApJ, 509, 283

Gómez, J. F., Sargent, A. I., Torrelles, J. M., et al. 1999, ApJ, 514, 287

Grady, C. A., Devine, D., Woodgate, B., \& Kimble, R. 1999a, AAS, 195, \# 02.08

Grady, C. A., Polowski, E. F., Henning, Th., et al. 2001, AJ, 122, 3396

Grady, C. A., Woodgate, B., Bruhweiler, F. C., et al. 1999b, ApJ, 523, L151

Green, S. 1975, ApJ, 201, 366

Green, S. 1989, ApJS, 70, 813

Green, S. 1991, ApJS, 76, 979

Green, S. 1994, ApJ, 434, 188

Green, S. 1995, ApJS, 100, 213

Green, S., \& Chapman, S., 1978, ApJS, 37, 169

Green, S., \& Thaddeus, P. 1974, ApJ, 191, 653

Greenhill, L. J., Gwinn, C. R., Schwartz, C., Moran, J. M., \& Diamond, P. J. 1998, Nature, 396, 650

Guilloteau, S., Delannoy, J., Downes, D., et al. 1992, A\&A, 262, 624

Haas, M., Leinert, Ch., \& Lenzen, R. 1992, A\&A, 261, 130

Hunter, T. R., Testi, L., Zhang, Q., \& Sridharan, T. K. 1999, AJ, 118, 477

Hartmann, L. 1998, in Accretion Processes in Star Formation, (Cambridge, UK, New York, Cambridge University Press), 53

Hasegawa, T. I., \& Mitchell, G. F. 1995, ApJ, 441, 665

Harvey, P. M., Campbell, M. F., Hoffman, W. F., Thronson, H. A., \& Gately, I. 1979, ApJ, 229, 990
Helmich, F. P. 1996, Ph.D. Thesis, Leiden Observatory

Helmich, F. P., \& van Dishoeck, E. F. 1997, A\&AS, 124, 205

Helmich, F. P., van Dishoeck, E. F., Black, J. H., et al. 1996, A\&A, 315,173

Henning, Th., Schreyer, K., Launhardt, L., \& Burkert, A. 2000, A\&A, 353,211

Henning, Th., Pfau, W., \& Altenhoff, W. J. 1990, A\&A, 227, 542

Henning, Th., Friedemann, C., Gürtler, J., \& Dorschner, J. 1984, AN, 305, 67

Hoare, M. G., Glindemann, A., \& Richichi, A. 1996, Near-IR Speckle Imaging of Luminous Young Stellar Objects, in The Role of Dust in the Formation of Stars, ESO Proc., ed. H. U. Käufl, \& R. Siebenmorgen (Springer-Verlag, Berlin, Heidelberg, New York), 35

Hoare, M. G. 2001, private communication

Hodapp, K.-W. 1994, ApJS, 94, 615

Hogerheijde, M., \& van der Tak, F. F. S. 2000, A\&A, 362, 697

Hollenbach, D., Johnstone, D., Lizano, S., \& Shu, F. 1994, ApJ, 428, 654

Jäger, C., Mutschke, H., Dorschner, J., \& Henning, Th. 1998, A\&A, 332, 291

Joyce, R. R., Capps, R. W., Gillett, F. C., et al. 1977, ApJ, 312, L127

Kawabe, R., Ogawa, H., Fukui, Y., et al. 1984, ApJ, 282, L73

Kawabe, R., Hasegawa, T. I., Hayashi, S. S., \& Kaifu, N. 1987, Molecular Line Studies of the Bipolar Flow Source GL:490, in Star Forming Regions, ed. M. Peimbert, \& J. Jugaku (Reidel, Dordrecht), IAU Symp., 115, 352

Koerner, D. W., \& Sargent, A. I. 1995, AJ, 109, 2138

Kutner, M. L., \& Ulich, B. L. 1981, ApJ, 250, 341

Lada, C. J., \& Harvey, P. M. 1981, ApJ, 245, 58

Lahuis, F., \& van Dishoeck, E. F. 2000, A\&A, 355, 699

Laughlin, G., \& Bodenheimer, P. 1994, ApJ, 436, 335

Lemke, D., Klaas, U., Abolins, J., et al. 1996, ISO special issue, A\&A, 315, L64

Manske, V., Henning, Th., \& Men'shchikov, A. B. 1998, A\&A, 331, 52

Mannings, V., \& Sargent, A. I. 1997, ApJ, 490, 792

Mannings, V., \& Sargent, A. I. 2000, ApJ, 529, 391

Mathis, J. S., Rumpl, W., \& Nordsieck, K. H. 1977, ApJ, 215, 425

McCaughrean, M. J., Stapelfeldt, K. R., \& Close, L. M. 2000, High-Resolution Optical and Near-Infrared Imaging of Young Circumstellar Disks, in Protostars and Planets IV, ed. V. Mannings, A. Boss, \& S. S. Russell (Univ. of Arizona Press, Tuscon), 485

Men'shchikov, A. B., \& Henning, Th. 1997, A\&A, 318, 879

Minchin, N. R., Hough, J. H., Burton, M. G., \& Yamashita, T. 1991, MNRAS, 251, 522

Minier, V., Booth, R. S., \& Conway, J. E. 2000, A\&A, 362, 1093

Mitchell, G. F., Hasegawa, T. I., \& Schella, J. 1992, ApJ, 386, 604

Mitchell, G. F., Lee, S. W., Maillard, J.-P., et al. 1995, ApJ, 438, 794

Monteiro, T. S. 1985, MNRAS, 214, 419

Mozurkewich, D., Schwartz, P. R., \& Smith, H. A. 1986, ApJ, 311, 371

Mundy, L. G., \& Adelman, G. A. 1988, ApJ, 329, 907

Nakamura, A., Kawabe, R., Kitamura, Y., et al. 1991, ApJ, 383, L81

Narayanan, G., \& Walker, C. K. 1996, ApJ, 466, 844

Natta, A., Grinin, V. P., \& Mannings, V. 2000, Properties and Evolution of Disks around Pre-Main-Sequence Stars of Intermediate Mass, in Protostars and Planets IV, ed. V. Mannings, A. Boss, \& S. S. Russell (Univ. of Arizona Press, Tuscon), 559

Natta, A., Grinin, V. P., Mannings, V., \& Ungerechts, H. 1997, ApJ, 491,885

Ossenkopf, V., \& Henning, Th. 1994, A\&A, 291, 943 
Palla, F., \& Stahler, S. W. 1992, ApJ, 392, 667

Pantin, E., Waelkens, C., \& Lagage, P. O. 2000, A\&A, 361, L9

Persson, S. E., McGregor, P. J., \& Campbell, B. 1988, ApJ, 326, 339

Plambeck, R. L., Wright, M. C. H., Welch, W. J., et al. 1982, ApJ, 259, 617

Plume, R., Jaffe, D. T., \& Evans II, N. J. 1992, ApJS, 78, 505

Preibisch, Th., Ossenkopf, V., Yorke, H. W., \& Henning, Th. 1993, A\&A, 279, 577

Rieke, G. H., \& Lebofsky, M. J. 1985, ApJ, 288, 618

Sandell, G. 1994, MNRAS, 271, 75

Schinke, R., Engel, V., Buck, U., Meyer, H., \& Diercksen, G. H. F. 1985, ApJ, 299, 939

Schreyer, K., Helmich, F. P., van Dishoeck, E. F., \& Henning, Th. 1997, A\&A, 326, 347

Schreyer, K., Henning, Th., \& Wiesemeyer, H. 1999, High-resolution submm/mm interferometry of the Orion-KL region, in The Orion Complex Revisited, ed. M. J. McCaughrean, ASP Conf. Ser., 999

Schreyer, K., Henning, Th., Stecklum, B., \& Wiesemeyer, H. 2002, A\&A, in prep.

Schröder, K., Stämmler, V., Smith, M. D., Flower, D. R., \& Jaquet, R. 1991, J. Phys. B, 24, 2487

Scoville, N. Z., Kleinmann, S. G., Hall, D. N. B., \& Ridgway, S. T. 1983, ApJ, 275, 201

Shepherd, D. S., \& Kurtz, S. E. 1999, ApJ, 523, 690

Shepherd, D. S., Claussen, M. J., \& Kurtz, S. E. 2001, Science, 292, 1513

Shu, F. H., Tremaine, S., Adams, F. C., \& Ruden, S. P. 1990, ApJ, 358, 495

Simon, T., Simon, M., \& Joyce, R. R. 1979, ApJ, 230, 127

Simon, M., Righini-Cohen, G., Felli, M., \& Fischer, J. 1981a, ApJ, 245,552

Simon, M., Righini-Cohen, G., Fischer, J., \& Cassar, L. 1981b, ApJ, 251,552
Simon, M., Felli, M., Cassar, L., Fischer, J., \& Massi, M. 1983, ApJ, 266, 623

Snell, R. L., Scoville, N. Z., Sanders, D. B., \& Erickson, N. R. 1984, ApJ, 284, 176

Stahler, S. W., Palla, F., \& Ho, P. T. P. 2000, The Formation of Massive Stars, in Protostars and Planets IV, ed. V. Mannings, A. Boss, \& S. S. Russell (Univ. of Arizona Press, Tuscon), 327

Stone, J. M., Gammie, C. F., Balbus, S. A., \& Hawley, J. F. 2000, Transport Processes in Protostellar Disks, in Protostars and Planets IV, ed. V. Mannings, A. Boss, \& S. S. Russell (Univ. of Arizona Press, Tuscon), 589

Thompson, R. I. 1984, ApJ, 283, 165

Thompson, R. I., \& Tokunaga, A. T. 1979, ApJ, 231, 736

Tielens, A. G. G. M., Tokunaga, A. T., Geballe, T. R., \& Baas, F. 1991, ApJ, 381, 181

Turner, B. E. 1990, ApJ, 362, L29

van den Ancker, M. E., Wesselius, P. R., \& Tielens, A. G. G. M. 2000, A\&A, 355, 194

van der Tak, F. F. S., \& van Dishoeck, E. F. 2000, A\&A, 358, L79

van der Tak, F. F. S., van Dishoeck, E. F., Evans II, N. J., Bakker, E. J., \& Blake, G. A. 1999, ApJ, 522, 991

van der Tak, F. F. S., van Dishoeck, E. F., \& Caselli, P. 2000a, A\&A, 361,327

van der Tak, F. F. S., van Dishoeck, E. F., Evans II, N. J., \& Blake, G. A. 2000b, ApJ, 537, 283

Vogel, S. N., Bieging, J. H., Plambeck, R. L., Welch, W. J., \& Wright, M. C. H. 1985, ApJ, 296, 600

Willner, S. P., Gillett, F. C., Herter, T. L., et al. 1982, ApJ, 253, 174

Wilson, T. L., \& Rood, R. T. 1994, ARA\&A, 32, 191

Yamashita, T., Sato, S., Nadata, T., et al. 1989, ApJ, 336, 832

Yorke, H. W., Bodenheimer, P., \& Laughlin, G. 1995, ApJ, 443, 199

Zhang, Q., Hunter, T. R., \& Sridharan, T. K. 1998, ApJ, 505, L151 